Research Square

Preprints are preliminary reports that have not undergone peer review. They should not be considered conclusive, used to inform clinical practice, or referenced by the media as validated information.

\title{
Sociodemographic, health and fracture profiles of a 4-year cohort of 266,324 first incident upper extremity fractures in Ontario.
}

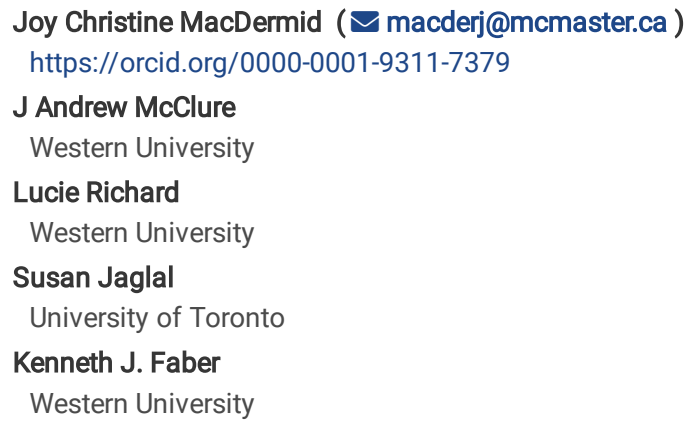

\section{Research article}

Keywords: fractures, epidemiology, upper extremity, distal radius, proximal humerus, social deprivation, comorbidity, nerve injury, tendon injury, sex differences, scaphoid

Posted Date: January 2nd, 2020

DOI: https://doi.org/10.21203/rs.2.20050/v1

License: (c) (1) This work is licensed under a Creative Commons Attribution 4.0 International License. Read Full License 


\section{Abstract}

Background

The purpose of this study was to describe 1 st incident fractures of the upper extremity in terms of fracture characteristics, demographics, social deprivation and comorbid health profiles.

Methods:

Cases with a 1st adult upper extremity fracture from the years 2013 to 2017 were extracted from administrative data in Ontario, (population 14.3M). Fracture locations (ICD-10 codes) and associated characteristics (open/closed, associated hospitalization within 1-day, associated nerve or tendon injury) were described by fracture type, age category and sex. Fracture comorbidity characteristics were described in terms of the prevalence of diabetes, rheumatoid arthritis; and the Charlson Comorbidity Index. Social marginalization was expressed using the Ontario Marginalization Index (ON-Marg) for material deprivation, dependency, residential instability, ethnic concentration.

Results

From 266,324 first incident UE fractures occurring over 4 years, $51.5 \%$ were in women and $48.5 \%$ were in men. This masked large differences in age-sex profiles. Most commonly affected were the hand (93K), wrist/forearm( $80 \mathrm{~K})$, shoulder (48K) or elbow (35K). The highest number of fractures: distal radius (DRF, 47.4K), metacarpal (30.4K), phalangeal (29.9K), distal phalangeal (24.4K), proximal humerus (PHF, 21.7K), clavicle (15.1K), radial head (13.9K), and scaphoid fractures (13.2K). The most prevalent multiple fractures included: multiple radius and ulna fractures (11.8K), fractures occurring in multiple regions of the upper extremity $(8.7 \mathrm{~K})$, or multiple regions in the forearm (8.4K). Fractures most common in 18 - 40-year-old men included metacarpal and finger fractures. A large increase in fractures in women over the age of 50 occurred for: DRF, PHF and radial head. Tendon $(0.6 \%$ overall; $8.2 \%$ in multiple finger fractures) or nerve injuries ( $0.3 \%$ overall, $1.5 \%$ in distal humerus) were rarely reported. Fractures were open in $4.7 \%$, highest for distal phalanx ( $23 \%$ ). Diabetes occurred in $15.3 \%$, highest in PHF (29.7\%). Rheumatoid arthritis occurred more commonly in women ( $2.8 \%$ vs $0.8 \%$ men). The Charlson Index indicated low comorbidity (mean=0.2; median=0: $2.4 \% 3+$ ), highest in PHF (median=0; $6.6 \% 3+$ ). Higher fracture burden was related to instability (excess of fractures in lower 2 quartiles $4.8 \%$ ), although social indices varied by fracture type.

Conclusions

Fracture specific prevention strategies should consider fracture-specific age-sex interactions, health, behavioural and social risks

\section{Introduction}

Clinicians and health systems need to be prepared to manage the large volume of upper extremity fractures that present for care. Some upper extremity fractures like distal radius fractures (DRF) and proximal humerus fractures (PHF) are classified as fragility fractures and considered an early indicator of compromised bone health[1]. Others are minor injuries that cause temporary pain and disability, and then recover optimally with minimal future risk. Understanding the volumes and profiles of different fracture types can inform management. The profiles of people incurring a first fracture is likely to be different from those with recurrent fractures.

Previous studies have focused on the epidemiology of the more common upper extremity fractures [2] particularly wrist [3], hand [4] and proximal humerus fractures [5,6], while other upper extremity fractures have been rarely described. None of these studies have focused on a first presenting fracture. DRF are so common that $6 \%$ of males and $33 \%$ of females $[2,3]$ will have one at some point in their lifetime. The interest in DRF is partially related to the fact they can be an early indicator of future fragility fractures[4]. A systematic review of DRF epidemiology examined nine publications addressing secondary risk, that concluded that a prior wrist fracture was associated with approximately a doubling of risk for subsequent fractures[5]. Increasing rates of DRF in both working ages and older adults[6], is a concern for health care cost containment. Hand fracture rates have also been described, and the burden relates to costs and occupational impacts[7, 8]. Proximal humeral fractures (PHF) are less frequent[1,5], but they can indicate compromised bone health[4] and fragility or frailty[9]. Frailty has been associated with greater risk of fracture, disability, and falls in women aged 55 and older in 10 countries [10].

A few studies that have investigated the epidemiology of upper extremity fracture have provided partial information since they provide data on only a few fracture types[11], sampled in only one city[12], only included patients over 60 or $65[13,14]$, excluded trauma[14] or have not excluded people with a prior upper extremity fracture. A detailed study of upper extremity fractures based on emergency and inpatient cases from 8 representative states in the United States, included insured and uninsured persons and defined fracture rates based on ICD-9 codes [15]. DRF had the highest incidence at 16.2 per 10,000 person years, followed by hand (phalangeal and metacarpal 12.5 and 8.4 respectively), PHF (6.0) and clavicle (5.8) fractures. Many fracture epidemiology studies focus solely on rates and not on the profiles of different fracture types. The description of $1^{\text {st }}$ incident fractures is important, since this is the first opportunity to evaluate if future fractures can be prevented, and once recurrent fractures are present this represents a different, potentially clearer, risk profile.

Although clinical studies often consider associated comorbid health and associated injuries as important, few epidemiologic studies have defined these profiles in large cohorts. One epidemiologic study of traumatic nerve injury found that humeral and ulnar fractures were the most common reason for upper extremity nerve injury[16], but studied cases of nerve injury not the rates or proportions within fractures. Nerve injuries can be potentially life-changing, may require surgery and extensive rehabilitation which can be more complicated than the fracture care. Understanding the frequency and distribution of these associated injuries is important.

Therefore, the purposes of this study were to describe a cohort of adult patients having their first upper extremity fracture in terms of: 
1. Injury patterns: fracture numbers, fracture subtypes (location, \% open), associated nerve or tendon injuries, injury seasonal patterns and hospitalization rates

2. Comorbid health status: overall comorbidity (Charlson comorbidity, presence of diabetes, rheumatoid arthritis)

3. Sociodemographic profiles (age, sex/gender, income quintile, rural/urban status, material deprivation, dependency, residential instability, and ethnic concentration).

\section{Methods}

Design: Descriptive analysis of a cohort of 1 st upper extremity fractures identified using administrative data

Data Sources and Extraction

Data Extraction and Sources

ICES houses linkable and anonymized health and demographic databases. Under Ontario law (Personal Health Information Protection Act, Ontario Regulation 329/04), ICES can receive and use health information without consent for the purposes of compiling and analyzing statistical information after users have completed rigorous procedures for privacy protection. ICES is a prescribed entity partially funded by the Ministry of Health and Long-Term Care (single insurer for the province's population of 14.3 million patients). Exclusions include people under Federal jurisdiction (e.g. First Nations living on reserve, full-time members of the Canadian Armed Forces, individuals in the Royal Canadian Mounted Police and inmates from federal correctional facilities).

Patient data were extracted from relevant administrative data sources that arise from hospital admission/discharge (Discharge Abstract Database), outpatient surgery (Same Day Surgery), emergency visits (National Ambulatory Care Reporting System), physician billing data (Ontario Health Insurance Program [OHIP] database), and general use data sets containing sociodemographic data (Registered Persons Database). ICES-developed disease cohorts were used to identify rheumatoid arthritis (Ontario Rheumatoid Arthritis Database) and diabetes (Ontario Diabetes Database). Data were linked to associated demographic and fracture information using unique, encoded identifiers, and analyzed at ICES Western. Data were depersonalized or aggregated for confidentiality reasons, and precautions were taken to ensure indirect identification was not possible (e.g. suppressing small cells).

Fracture Identification/Description.

This retrospective cohort study includes all adult patients who visited the emergency room with an upper extremity fracture from January 1,2013 to December 31. 2017. Only the first such event was eligible for inclusion. To ensure all fracture-related diagnoses were captured, we also included fractures reported during ER visits and hospital admissions within 5 days of the initial ER visit. Fractures coded as 'questionable' were counted if there was other supporting evidence of fracture from an ER visit, hospitalization, or OHIP record. In addition to data cleaning exclusions, we excluded non-Ontario residents and those with evidence of an upper extremity fracture, as an adult, within the previous 10-years (previous pediatric fractures were permitted). To help make sure we identified all previous fractures, we also excluded individuals who were not eligible for healthcare services in Ontario for this full 10-year look-back period.

Fracture profiles

Fractures were described by location and type (open/closed), season and whether a tendon or nerve injury was identified. Fracture codes were grouped together based on clinical relevance; compiling open and closed fractures, and codes where multiple codes were used for the same type: clavicle, scapula, proximal humerus, humerus shaft, distal humerus, head/neck of the radius, distal radius fractures, carpal bones other than the scaphoid, or 1 st metacarpal (ICD-10 codes multiple areas on the 1st metacarpal but does not differentiate other metacarpals), proximal/other phalangeal fractures. Other specific fractures were identified by single codes including olecranon, coronoid, Monteggia, ulnar shaft, scaphoid, and distal phalanx fracture (but combined across open and closed options). Codes describing multiple concurrent fractures of the shoulder, radius and ulna, forearm, metacarpals or digits were combined and identified as multiple fractures within those areas. Where concurrent fractures codes were from different regions (e.g. shoulder and hand), this was coded as fracture in $>1$ region.

Nerve injury was grouped as a major nerve injury when codes for specific major nerves in the upper arm (ulnar, median, radial, axillary, musculocutaneous), forearm (median, ulnar, radial), or wrist (median/ulnar) were identified; and as any nerve injury by collapsing all specific and non-specific nerve codes for each of these 3 areas. Hospitalization within 1 day was extracted.

Patient Profiles

A descriptive profile of the entire cohort and major fracture subtypes was constructed to describe demographics, fracture characteristics, comorbidity and social marginalization; considering age and gender profiles for specific fractures. Age was classified based on clinically relevant subgroups: aged 18 to 40 (young adults), 41 to 50 (younger middle-age adults), 51 to 65 (older middle-age), 66 to 80 (older adults), $81+$ (very old adults). The age groups were selected to represent anticipated different health and fracture risk profiles, with young adults having the best bone health and being more likely to engage in higher fracture risk activities. Younger middle-aged adults were considered as being transitional in terms of activity and bone health, while older middle-age individuals (51-65 years) are those with emerging fracture risks. Older adults were considered more likely to have associated comorbidities and the very old to have a higher probability of frailty.

Diabetes (from ODD) and Rheumatoid Arthritis (from ORAD) diagnoses were identified using ICES defined cohorts. People with diabetes were identified as all individuals in the fracture cohort who appeared in the ODD before the fracture index date, based on having at least one hospitalization or two physicians' 
service claims for diabetes within a 2-year period. The ODD has high sensitivity (86\%) and specificity (97\%) for identifying diabetes when compared to extraction from primary care charts[17]. The RA cohort algorithm in ORAD has high sensitivity and specificity compared to a rheumatologist diagnosis[18, 19].

We attempted to extract data on osteoporosis. However, since a 1st upper extremity fracture at the age of 50 or older may initiate the diagnostic process, Bone Mineral Density data was rarely available. Since this data is used in definition of osteoporosis, we suspected underreporting.[20] Similar problems arose in extracting an osteoarthritis diagnosis, since this is not consistently coded unless it is the primary reason for the visit. Given validity concerns with these 2 diagnoses, we did not report osteoporosis or osteoarthritis comorbidity, despite their importance.

Overall comorbidity indicators were derived from the Charlson comorbidity index[21]. The Charlson Comorbidity Index categorizes comorbidity using International Classification of Diseases (ICD) diagnosis codes. Each comorbidity category has an associated weight (from 1 to 6 ), based on the adjusted risk of mortality, and the sum of all the weights results in a single comorbidity score for a patient[21, 22]. A score of zero indicates that no comorbidities were found. The Charlson Index is the most extensively studied comorbidity index for predicting mortality[23].

The Ontario Marginalization Index (ON-Marg) was used to assess social inequities (material deprivation, dependency, residential instability, ethnic concentration)[24]. ON-Marg was developed using a theoretical framework based on previous work on deprivation and marginalization and empirically derived using principal component factor analysis. A quintile score is associated with geographic areas, so that each quintile represents $20 \%$ of the reference population. To describe marginalization, we compared the top two quintile values to the bottom 2 quintiles. We graphed the size and direction of any excess of fractures in the lower double-quintile (expected 40\%). For the ON-Marg Index, those in lower quartiles are considered more deprived or more marginalized. Maternal deprivation reflects socioeconomic status, education and single-parent families and is comprised of indices including the proportion without a high school diploma, single-parent families, proportion of income from government support, proportion of those over 15 who are unemployed, proportion classified as low income and the proportion living in houses classified as requiring major repair. Ethnic concentration reflects the proportion of the neighborhoods who are recent immigrants or visible minorities. Dependency reflects the extent to which the population is not supported by employment income and includes indicators of proportion of people over 65 , a ratio of younger to older people, and the proportion not participating in the workforce. Residential instability reflects home security, ownership and occupancy and is comprised of indicators of living alone, proportion of adults, number of people per dwelling, proportion who are single/divorced/widowed, and the proportion who have moved in the past 5 years. Marginalization and co-morbidity have previously been associated using ICES data[25].

Analysis and hypotheses

Our purposes were descriptive and the analysis focused on counts or percentages that described the number of fractures by type and disaggregated by gender and age groups; describing profiles in terms of the demographics, fracture characteristics, comorbidity and social indicators. We expected that young adults would be most susceptible to higher energy "misfortune" fractures; whereas the older middle-aged cohort would contain both active and compromised individuals. We expected gender differences in young adults to reflect higher risk-taking or involvement in fracture susceptible activities in young men. We expected sex differences in bone health to emerge by the 50-year-old age category and to be more pronounced in fractures that are considered fragility fractures such as DRF and PHF. We expected that frailty fractures would be prevalent in the old and very old subgroups. We considered differences in social deprivation of $5 \%$ between upper and lower categories to be potentially relevant and $10 \%$ or more very likely to be relevant.

\section{Results}

After exclusions from the potential cohort $(n=506,071)$, mostly related to pediatric fractures $(n=174,378)$, we were left with a cohort of 266,324 people with a first upper extremity fracture occurring over a four-year interval $(n=44,236$ were excluded because they had prior fractures) (Fig. 1$)$. Overall, looking grossly at the fracture distributions by region the most common fracture sites were the hand (94K), followed by the wrist (80K), shoulder (49K) and elbow (35K) - Fig. 2. The most common shoulder fractures were PHF and clavicle (Fig. 3 overall; Figs. 9-12). The most common elbow fracture was a radial head fracture (Fig. 4 overall; Figs. 13-14). DRF represented about one half of the wrist and forearm fractures with scaphoid being the next most prevalent (Fig. 5 overall Figs. 1516)). Hands had high volumes of fractures of the metacarpals, distal phalanges and other phalangeal codes (Fig. 6 overall, Figs. 17-19 subtypes). When looking at specific fracture subtypes across regions (Table 1 for complete data) the most common were in order: distal radius fractures (DRF) Fig. 15, metacarpals (Fig. 17), phalangeal fractures (Fig. 18), distal phalangeal fractures (Fig. 19), proximal humerus fractures (PHF) Fig. 11, clavicle fractures (Fig. 10), radial head fractures (Fig. 13), and scaphoid fractures (Fig. 16). The next most prevalent codes were for multiple fractures (Figs. 20-22) including, by volume, multiple radius and ulna fractures (Fig. 22), fractures occurring in multiple regions of the upper extremity (Fig. 20), or multiple regions in the forearm. See Table 1. Uncommon fractures included coronoid $(n=327)$, isolated radius shaft $(n=216)$, isolated ulnar shaft $(n=170)$ and Monteggia $(n=25)$. 
Table 1

Demographics of the sample across different upper extremity fractures

\begin{tabular}{|c|c|c|c|c|c|c|c|c|}
\hline \multicolumn{9}{|l|}{ Demographics } \\
\hline \multirow[t]{2}{*}{ Fracture Type } & \multirow[t]{2}{*}{$\mathrm{n}$} & \multicolumn{3}{|l|}{ Age (years) } & \multicolumn{2}{|l|}{ Sex (\%) } & \multicolumn{2}{|c|}{ Location (\%)* } \\
\hline & & Mean (SD) & Median & IQR & Women & Men & Urban & Rural \\
\hline DRF & 47,407 & $59.3(18.5)$ & 60 & $49-72$ & 73.3 & 26.7 & 82.7 & 16.1 \\
\hline MC 2-4 & 30,462 & $37.9(18.2)$ & 32 & $23-49$ & 29.5 & 70.5 & 82.1 & 16.1 \\
\hline Phalanx & 29,912 & $44.6(18.5)$ & 44 & $29-57$ & 42.6 & 55.4 & 82.1 & 16.6 \\
\hline DP & 24,407 & $45.1(17.1)$ & 45 & $31-57$ & 28.9 & 71.1 & 77.6 & 21.1 \\
\hline PHF & 21,765 & $68.5(15.7)$ & 69 & $59-81$ & 73.9 & 26.1 & 82.6 & 16.2 \\
\hline Clavicle & 15,121 & $50.6(21.6)$ & 50 & $31-66$ & 34.1 & 64.9 & 79.9 & 17.9 \\
\hline Radial head & 13,934 & $46.5(17.9)$ & 47 & $31-59$ & 60.2 & 39.8 & 86.3 & 12.8 \\
\hline Scaphoid & 13,279 & 44.0 (19.7) & 42 & $26-59$ & 50.5 & 49.5 & 85.7 & 13.3 \\
\hline Multiple Radius and Ulna & 11,844 & $57.0(20.0)$ & 56 & $44-72$ & 66.4 & 33.6 & 84.0 & 14.4 \\
\hline Multiple UE regions & 8697 & $55.6(21.1)$ & 57 & $39-72$ & 56.8 & 43.2 & 79.4 & 18.9 \\
\hline Forearm multiple & 8372 & $54.7(20.2)$ & 56 & $36-69$ & 61.7 & 38.3 & 78.2 & 19.7 \\
\hline Olecranon & 3707 & $58.2(20.8)$ & 59 & $44-75$ & 52.3 & 47.7 & 84.8 & 14.2 \\
\hline Scapula & 3255 & 56.7 (19.7) & 57 & $43-72$ & 32.7 & 67.3 & 78.7 & 19.7 \\
\hline Entire cohort & 266,324 & $51.5(20.8)$ & 53 & $33-67$ & 51.5 & 48.5 & 81.9 & 16.6 \\
\hline
\end{tabular}

The mean age of the cohort was 51.5 years and was slightly female dominant (51.5\%) (Table 1). When examining the different demographic profiles of major fracture subtypes, PHF demonstrated the oldest mean age (68.5) and metacarpal fractures the youngest age (37.9) (Table 1, Fig. 11). The largest number of fractures occurred in 18 to 40-year-olds for metacarpal, phalangeal, clavicle, radial head, and scaphoid fractures (Figs. 17, 19, 10, 13, 16). Clavicle fractures (Fig. 10) and radial head fractures (Fig. 13) while presenting in larger numbers for the 18 to 40 -year-olds, also had substantial proportions in the 51 to 65 -yearold group. For DRF (Fig. 15) and multiple radial/ulnar fractures (Fig. 22), the largest proportion of fractures occurred in the 51-65 group; while in PHF the largest number of fractures number occurred in the 66-80 subgroup. The fractures that were the most female predominant were PHF (73.9\%) and DRF (73.3\%) (Table 1). The fractures that were most male predominant were distal phalangeal fractures (71.1\%) and metacarpal fractures (70.5\%) (Table 1).

Examining age groups for men versus women for specific fractures provided further insight that sex/gender modifies the age patterns for specific fractures. For DRF (Fig. 15), there is a large excess in terms of women presenting with a first DRF starting with the 51-65-year-old age category and continuing at older ages. For metacarpal and phalanx fractures (Figs. 17 and 18) there is a substantial male excess of fractures in the 18 to 40 -year-olds, which is less substantial in the middle-aged categories and reverses so that there is a small excess of women after the age of 66. For the distal phalanx (Fig. 19), a male predominance is present in all age groups, although this is most predominant in the 18 to 40 -year-olds. In the oldest group, few DP fractures were recorded, but there is a small excess of women. For PHF (Fig. 11), a small number of fractures and a small excess in males present in the 18 to 40 -year-old group; thereafter, the excess is in women with a marked increase in the numbers of fractures and the female to male differential starting at the 51 to 65 -year-old age group. For clavicle fractures (Fig. 10), many fractures occur in the 18 to 40-year-old group with a substantial excess in males. An excess in men occurs throughout the lifespan until the 50-65-year-old group. Thereafter in the 66-80-year-old age group the numbers of clavicle fractures become equivalent across men/women and at $81+$, there is a greater number of women presenting with clavicle fractures. For radial head fractures (Fig. 13) there was an excess of men presenting in the 18 to 40-year-old group, thereafter more women were present in each age cohort; with the largest gender differential being observed in the 51-65-year-olds. For scaphoid fractures (Fig. 16), multiple fractures of the radius and ulna (Fig. 22), and humeral shaft fractures (Fig. 12) men were more prevalent in the youngest group (18-40) and thereafter women became more prevalent. More men than women presented with multiple shoulder fractures (Fig. 21), fractures in multiple regions of the UE (Fig. 20) and olecranon fractures (Fig. 14) in the two younger age groups; but thereafter women were more predominant. Men exceed women with respect to scapular fractures (Fig. 9) for all but the oldest age group.

Most of the patients were classified as coming from urban settings (81.9\%; rural $16.2 \%)$ Table 1 . The only fracture where the proportion of rurality exceeded $20 \%$ was for distal phalangeal fractures $(21.1 \%$ rural).

Overall, only $4.7 \%$ of fractures were coded as open fractures; and nerve $(0.3 \%)$ and tendon $(0.6 \%)$ injury was rarely reported (Table 2$)$. A higher proportion of nerve injuries was recorded for fractures in multiple regions (1.1\%) and phalangeal fractures (0.5\%). The fracture type that was most commonly recorded as an open fracture was a distal phalangeal fracture (23\%). In total $7.6 \%$ of the fractures were associated with a hospital admission. Hospital admission rates were highest for fractures occurring in multiple upper extremity regions (37.2\%) and olecranon fractures $(26.2 \%)$. 
Table 2

Injury characteristics of different upper extremity fractures

\begin{tabular}{|c|c|c|c|c|c|c|c|c|c|}
\hline \multicolumn{10}{|l|}{ Fracture Characteristics } \\
\hline \multirow[t]{2}{*}{ Fracture Type } & \multirow[t]{2}{*}{$\mathrm{n}$} & \multirow[t]{2}{*}{ \% Open } & \multirow[t]{2}{*}{ Nerve Injury } & \multirow[t]{2}{*}{ Tendon Injury } & \multirow[t]{2}{*}{ Hospital Admission } & \multicolumn{4}{|c|}{ Injury date (\%/quarter) } \\
\hline & & & & & & J-M & A-J & Ju-S & O-D \\
\hline DRF & 47,407 & 0.7 & 0.1 & 0.1 & 4.7 & 30.8 & 21.5 & 23.8 & 24.0 \\
\hline MC 2-4 & 30,462 & 1.3 & 0.1 & 0.3 & 1.1 & 22.3 & 25.4 & 28.5 & 23.8 \\
\hline Phalanx & 29,912 & 8.6 & 0.5 & 1.8 & 1.3 & 21.1 & 26.3 & 28.6 & 24.1 \\
\hline DP & 24,407 & 23 & 0.3 & 1.2 & 0.6 & 20.4 & 26.1 & 28.6 & 24.8 \\
\hline PHF & 21,765 & 0.3 & 0.1 & $\approx 0$ & 17.2 & 27.9 & 22.3 & 23.2 & 26.7 \\
\hline Clavicle & 15,121 & 0.6 & $\approx 0$ & $\approx 0$ & 12.3 & 20.0 & 25.7 & 32.9 & 21.1 \\
\hline Radial head & 13,934 & 0 & $\approx 0$ & $\approx 0$ & 2.0 & 22.2 & 25.8 & 30.0 & 22.0 \\
\hline Scaphoid & 13,279 & 0.2 & $\approx 0$ & 0.2 & 0.8 & 24.4 & 24.7 & 27.6 & 23.2 \\
\hline Multiple Radius and Ulna & 11,844 & 3.7 & 0.3 & 0.1 & 13.2 & 26.6 & 23.3 & 26.2 & 23.9 \\
\hline Multiple UE regions & 8697 & 9.2 & 1.1 & 0.7 & 37.2 & 21.5 & 24.3 & 30.5 & 23.8 \\
\hline Forearm multiple & 8372 & 1.8 & 0.2 & 0.2 & 9.2 & 25.9 & 24.3 & 26.9 & 22.9 \\
\hline Olecranon & 3,707 & 4.9 & 0.2 & $\approx 0$ & 26.2 & 28.4 & 21.3 & 25.7 & 24.6 \\
\hline Scapula & 3255 & 0.5 & $\approx 0$ & $\approx 0$ & 21.9 & 23.9 & 23.8 & 28.9 & 23.3 \\
\hline Entire cohort & 266,324 & 4.7 & 0.3 & 0.6 & 7.6 & 24.7 & 24.2 & 27.3 & 23.9 \\
\hline
\end{tabular}

Overall, the summer season had the highest proportion of fractures (27.3\%) (Table 2). Fractures that occurred most commonly in the winter (>28\% occurring between January and March) were DRF, PHF, and olecranon fractures. Fractures that had a higher predominance in the summer ( $>28 \%$ occurring between July and September) included: clavicle, radial head, fractures in multiple regions of the upper extremity, and distal phalangeal fractures.

Overall, the cohort of patients with a 1st upper extremity fracture represents a relatively healthy cohort as reflected in Charlson Index scores where $91.8 \%$ scored a grade 0 (mean 0.2) (Table 3). The proportion of cases with diabetes was $15.3 \%$ and RA was $1.8 \%$. Higher proportions of diabetes were seen in those with PHF (29.7\%) and DRF (19.9\%); or approximately 1 in 3 PHF and 1 in 5 DRF. Higher comorbidity on the Charlson index was reflected in higher mean scores for PHF (0.5), and higher proportions of individual who scored 3+: PHF (6.6\%), clavicle (3.4\%), multiple upper extremity regions (3.1\%) and olecranon fractures (3\%). The percentage of patients with diabetes for different fracture types is reported separately for men and women (Fig. 23). In PHF, DRF and olecranon fractures the percentage of patients with diabetes were higher for men; whereas for all other fractures the percentage with diabetes was higher for women. For all fracture types a greater percentage of women had RA (Fig. 23). 
Table 3

Fracture comorbidity of different upper extremity fractures

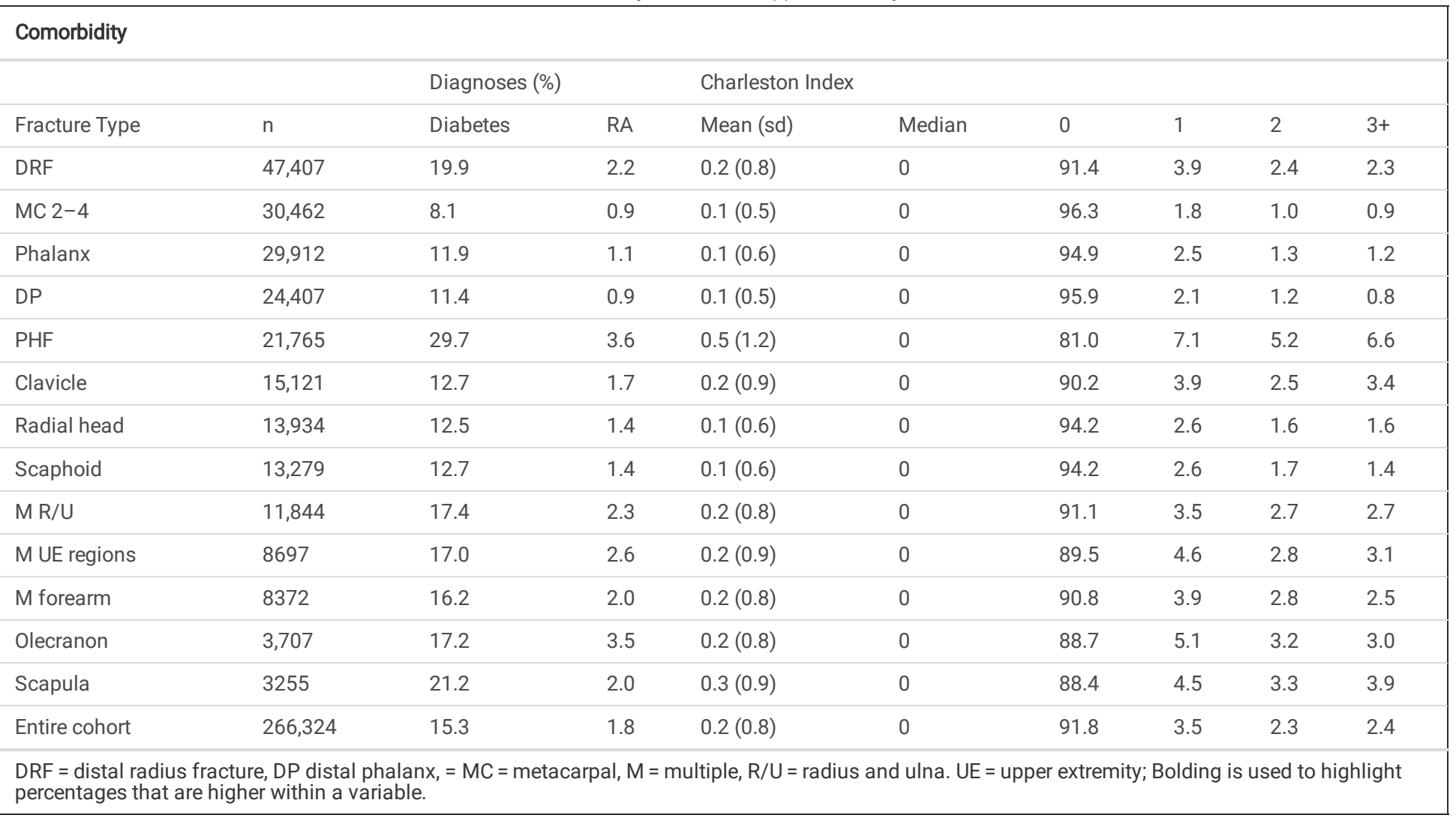

Table 4

Neighbourhood sociodemographics of different upper extremity fractures

\begin{tabular}{|c|c|c|c|c|c|c|c|c|c|c|c|}
\hline \multicolumn{12}{|c|}{ Neighborhood Sociodemographics } \\
\hline \multirow[b]{2}{*}{ Fracture Type } & \multirow[b]{2}{*}{$\mathrm{n}$} & \multicolumn{2}{|c|}{ Income } & \multicolumn{2}{|c|}{ Dependency } & \multicolumn{2}{|c|}{ Deprivation } & \multicolumn{2}{|c|}{ Ethnic Concentration } & \multicolumn{2}{|c|}{ Instability } \\
\hline & & $U$ & $\mathrm{~L}$ & $U$ & $\mathrm{~L}$ & $U$ & $\mathrm{~L}$ & $U$ & $\mathrm{~L}$ & $U$ & $\mathrm{~L}$ \\
\hline DRF & 47,407 & 39.4 & 40.7 & 36.9 & 39.2 & 41.4 & 37.9 & 39.8 & 39.3 & 35.8 & 44.2 \\
\hline MC 2-4 & 30,462 & 44.6 & 35.8 & 43.0 & 37.3 & 36.3 & 43.6 & 39.6 & 44.6 & 33.9 & 46.4 \\
\hline Phalanx & 29,912 & 40.2 & 39.4 & 43.1 & 37.1 & 40.2 & 39.5 & 37.6 & 42.7 & 35.6 & 44.8 \\
\hline DIP & 24,407 & 39.2 & 40.0 & 42.2 & 39.6 & 40.6 & 38.5 & 43.3 & 37.8 & 40.1 & 39.1 \\
\hline PHF & 21,765 & 43.6 & 36.9 & 31.3 & 50.6 & 39.0 & 41.2 & 43.3 & 36.0 & 29.9 & 50.5 \\
\hline Clavicle & 15,121 & 40.3 & 40.3 & 38.9 & 41.9 & 41.7 & 38.5 & 42.3 & 36.3 & 34.4 & 44.6 \\
\hline Radial head & 13,934 & 39.9 & 40.4 & 45.5 & 35.3 & 42.2 & 38.2 & 33.9 & 45.0 & 36.9 & 47.0 \\
\hline Scaphoid & 13,279 & 40.7 & 39.3 & 44.2 & 36.7 & 40.3 & 39.6 & 35.1 & 44.6 & 36.9 & 44.6 \\
\hline M R/U & 11,844 & 39.9 & 40.0 & 38.7 & 42.2 & 39.9 & 38.9 & 38.0 & 42.1 & 36.2 & 43.9 \\
\hline$M$ regions & 8697 & 41.5 & 38.8 & 36.2 & 44.7 & 39.7 & 39.8 & 43.4 & 35.7 & 34.3 & 45.0 \\
\hline M Forearm & 8372 & 42.6 & 38.1 & 36.8 & 43.8 & 39.2 & 40.0 & 43.6 & 35.6 & 32.9 & 46.2 \\
\hline Olecranon & 3,707 & 40.5 & 40.4 & 39.2 & 43.2 & 41.8 & 38.9 & 37.9 & 39.8 & 33.6 & 47.8 \\
\hline Scapula & 3255 & 42.6 & 38.2 & 36.8 & 43.8 & 39.2 & 40.0 & 43.6 & 35.6 & 32.9 & 36.2 \\
\hline Entire cohort & 266,324 & 41.2 & 39.0 & 39.5 & 41.2 & 39.9 & 39.8 & 39.9 & 39.7 & 35.3 & 44.8 \\
\hline
\end{tabular}

After lumping the upper 2 quartiles, and the lower 2 quartiles, we would have expected approximately $40 \%$ of the fractures to occur in either the upper or lower sociodemographic double-quartiles since these each reflect $40 \%$ of the population. Few remarkable excesses in fractures volumes were found with respect to neighbourhood income with the largest differential being for metacarpal fractures (44.6\% in upper; $35.8 \%$ in lower) (Table 4). For neighbourhood dependency, 
PHF had the most extreme differential between upper and lower categories with a large excess of fractures occurring in the more deprived category (31.3\% versus $50.6 \%$ ). For neighbourhood deprivation, findings were relatively unremarkable with the largest upper/lower differential occurring for metacarpal fractures (36.3\% vs $43.6 \%$ ). For neighbourhood ethnic concentration some fractures demonstrated an excess in the upper double-quartiles (PHF, multiple regions for multiple, clavicle); and other fractures demonstrated an excess in lower double-quartile (radial head and scaphoid). With respect to neighbourhood instability there was an overall excess of fractures in the lower (44.8\%) versus upper (35.3\%) double-quartiles, which was reflected across multiple fractures, and most predominantly in PHF (50.5\% lower versus $29.9 \%$ upper).

When marginalization was examined as a variance from the expected $20 \%$ in the most marginalized subgroup (lowest quintile) separately for men and women, some additional patterns emerged. Income, ethnicity and deprivation continued to demonstrate little impact on fracture prevalence for both men and women (Fig. 24). However, dependency had a large negative impact on fracture volumes for PHF on women (32.8\% versus expected $20 \%$ ), and this excess was present for men, but to lesser extent. For DRF and clavicle fractures, dependency was associated with an excess of fractures in women, but had little impact on men. Living in the most unstable neighbourhood was associated with an excess in most fractures' types, with one exception being DP fractures in men where a slightly lower than expected proportion occurred in the lowest quintile (18.1\% versus expected $20 \%)$. See Figs. 24 and 25 . Instability had more impact on women for DRF, clavicle, and phalangeal fractures; whereas, the excess in the lowest quintile was slightly higher for men in PHF and RH.

\section{Discussion}

This paper provides new information on the fracture, health, and social marginalization profiles of a large cohort of 1 st incident upper extremity fractures. Overall, the hand was the region most affected, reflecting high volumes of finger fractures. The overall ratio of women to men being $51.5 \%$ versus $48.5 \%$ masked dramatic differences in age-sex profiles that varied substantially across different fracture types; reinforcing the importance of considering sex and lifespan interactions. We selected age categories that reflected different bone health stages of the lifespan and found a variety of sex-lifespan fracture patterns. While it is commonly considered that DRF consists of a bi-modal distribution of young men and older women, we found that women were more prevalent in all age cohorts; although the differences were small in the 18-40-year-old age group. Conversely, PHF which are commonly considered a fragility fracture do have a small excess of men in the 18-40-year-old group; and the large excess of women is not evident until the 50-65 years subgroup. This pattern of inflection for women at 50 years of age was also observed for scaphoid fractures, radial head fractures, olecranon fractures, multiple fractures of the shoulder or multiple fracture of the entire upper extremity. This reversal of sex predominance between the young and older subgroups is thought to indicate different mechanisms of fracture, with younger people being likely to incur "misfortune" fractures that occur due to higher energy trauma, while fractures in older people are more likely to occur with low trauma when bone quality is compromised. Our data raises questions about which fracture subtypes beyond the commonly presumed fragility fractures (DRF and PHF) should be considered as potential indicators of compromised bone health, since similar inflections were observed across some fractures types not typically considered fragility fractures.

Some fractures including scapula, metacarpals, finger fractures and distal phalanx have a high preponderance in young men. These profiles may reflect differences in misfortune fractures which can arise due to greater participation in fracture-risk activities/behaviours e.g. contact sports, greater risk-taking, fights/aggressive actions, manual occupations, outdoor work etc. Gender differences in engagement in these exposures could explain the higher predominance of some fractures for young men. For example, metacarpal fractures have a high predominance amongst young men, but this differential disappears in older men, and as suggested by the common term "boxers' fractures" risk-taking, fights and aggressive behaviours might lead to elevated numbers of young men incurring these injuries. However, our data suggests this behaviour might dissipate with age, or at least that a 1st incident decreases with age (since recurrent fractures are not reported in this paper)line. Conversely, distal phalanx fractures have a substantial predominance in men throughout the lifespan, except at the oldest age group. The fact that these fractures are less affected by age may reflect the nature of the risk activities. We hypothesize that gender-role differences in occupational and recreational exposures including outdoors work, equipment use, and manual labour, contribute to lifelong finger fractures risk exposures in men. In the oldest age group, the number of distal phalanx fractures is higher in women, but this may reflect the greater longevity of women and a larger cohort of living women who can be affected. Our hypotheses cannot be tested, given the limitations of health service data. However, our hypotheses reflect the fact that sex and gender cannot easily be disarticulated when considering fracture epidemiology.

An important consideration when interpreting our data, particularly for the oldest age group is that the ratio between men and women in the population will vary over the life span and will become increasingly female predominant after the age of 80 . In Canada, at age 80 the ratio of women to men is approximately $4: 3$, at 85 it is $5: 3$, at 90 it is $2: 1$ and at 95 it is $4: 1$ [26]. Thus, the higher numbers of women having fractures, particularly in fractures that are male predominant at younger ages likely reflects the larger volume of women in the population. Since we examine fracture volumes and proportions, not rates from the existing population these differences should not be misinterpreted as an increased risk for older women in comparison to men, when looking at the data for the oldest age group.

Females might have lifelong elevated fracture risks due to their bone geometry and composition[27]. However, at age 50 we see a dramatic increase in DRF and PHF even though population numbers are very similar for men and women.[26] We expected sex differences in these fractures due to osteoporosis/osteopenia preferentially affecting females[27]. These fragility fractures typically occur with low trauma, such a fall from level ground. The sexage patterns in our PHF and DRF data concurs with findings from our mixed methods study where quantitative data from a cohort of more than 1400 patients with DRF[28] indicated that low trauma was more common in those over 45 years of age, and high trauma occurred most in the youngest (18-24-year-old) group. In the qualitative part of that study, we found the factors contributing to fractures included environment, risk taking behaviors, physical factors, and sports activities. Our data indicates that the excess of fractures in older female ( 50 + years) was also identifiable in scaphoid fractures, radial head fractures, multiple fractures of the radius and ulna, and humeral shaft fractures which are less often considered as fragility fractures. Future studies should investigate the extent to which these less common upper extremity fractures predict osteoporosis, and future hip and spine fractures-and whether the definitions of fragility fracture should be expanded. 
One advantage of administrative data is the potential to study rare diagnoses. Monteggia fractures are an example, where we hoped to gain novel information on this rare but challenging fractures. Since only 50 cases were reported in our four-year cohort, longer cohorts would be needed for future investigations. We suspect that underreporting may partially explain these low numbers, since not all Monteggia fractures might be identified by the specific code by nonspeciality physicians. Potentially these were classified as fracture dislocations rather than by their specific name. Our data suggest that a coding validation study would be needed prior to conducting any administrative data analyses on this particular fracture type.

We found very low rates of tendon of nerve injury. The overall rate was influenced by higher prevalence of nerve injury in finger and distal phalanx fractures; where the nerve injury is unlikely to cause permanent disablement. The higher proportion of $1.1 \%$ nerve injury seen in multiple fractures may relate to the severity of trauma and will likely have a more negative prognosis. Tendon injuries were also infrequently reported, and as expected were more common with finger fractures. Since no validation studies have been performed for nerve and tendon codes we cannot be confident that these estimates reflect accurate attribution of the nature and severity of tendon and nerve injuries. It is possible that some nerve and tendon injuries are recognized/coded later when upper extremity specialist surgeons become involved in the case. This should be investigated in a future longitudinal validation study.

Age standardized rates of RA in Ontario, using ICES data, are reported to be between $0.49-0.9 \%$ [29]. The overall rate of RA in our cohort of $1.8 \%$ might suggest an elevated fracture risk, although we only examined fractures cases and could not calculate relative risks or age-adjusted rates. Olecranon fractures, DRF and fractures in multiple regions had greater proportions of RA diagnoses. The fact that the percentage of RA diagnoses were higher in women than men throughout all fractures is consistent with the sex distribution of RA [29]. Diabetes was present in 15.3\% of the cohort; but elevated proportions were evident for olecranon fractures, DRF and PHF. ICES data have previously established that diabetes is associated with elevated risk of hip fractures in both men and women, even after controlling for age, greater comorbidity, being less likely to have had a BMD test, and more likely to be taking medications that increase risk of falling. [30] Another epidemiologic study found elevated fracture risk in patients with diabetes, for both upper and lower extremity fractures[31]. While overall the cohort with a 1st upper extremity fracture is relatively healthy, the prognosis of the subset with RA, diabetes, or elevated Charlson indices warrants further exploration, especially with respect to potential strategies to reduce secondary fractures.

One of the unique aspects of this study was the inclusion of indices of neighbourhood social deprivation which has been rarely studied as factor in fractures. Income had limited impact, with small excesses appearing in both upper and lower quartiles in certain fractures. PHF were the only type of fracture where a potentially relevant income effect was observed, which was an excess of fractures in the higher income double quartiles, for both sexes. Income may have been conflated with other more influential social indices. Therefore, our conclusion is similar to that suggested by Karl et al[15] who concluded that SES, which they based on income, was not substantially related to fracture rates. They reported an excess of metacarpal and phalangeal fractures in their low-income group, which we did not find. However, we did find this excess in the dependency social indicator which encompasses employment. This supports our hypothesis that gendered employment exposures may partially explain "sex" differences in hand fractures. Conversely, while the prior study did not report that income affected PHF volumes; we did find some effects. Age affects income indicators since older people with a PHF are more likely to be retired. The relationship between wealth, financial independence and income is variable between people, methods for calculating the SES-income indicators, and across cohorts. We focused on volumes not rates, and defined SES using multi-component indices which may have contributed to the minor differences in our findings in comparison to those reported by Karl et al who uses rates and a single indicator of income[15].

With respect to deprivation, the only potentially relevant difference was for metacarpal fractures where the cohort excess in the lower double-quartile was $6.3 \%$. When examining the sex-specific proportions in the lowest quartile by fracture, this effect was present in males, but not in females. This index includes the impact of single parent families which might suggest that lack of parental supervision, or having a father in the home, which may have a larger impact on young men engaging in fracture-risk behaviours than it does for young women. This hypothesis has been empirically demonstrated in children[32]. However, our data can only highlight areas of differences at the neighbourhood level and while they may warrant exploration, causation cannot be inferred.

There were multiple differences that were potentially relevant with respect to ethnic concentration. DP, PHF, clavicle, scapula and multiple fractures were more prevalent in less ethnic neighbourhoods; whereas metacarpal, phalanx fractures, radial head and scaphoid had an excess in more ethnic neighbourhoods. Ethnicity may affect fractures through multiple mechanisms including sex differences in genetic risks that affect the size and quality of bone, gender-based differences in behaviour norms that affect behaviours and life roles, and social differences that affect work and life exposures. African American and Hispanic men have been reported as having higher incidence and younger onset of hip fractures than white men[33], which is consistent with what we found for some upper extremity fractures, although not the classic fragility-subtypes.

Dependency was associated with some potentially relevant excesses in fractures, but the excess varied across fracture types. Metacarpal, phalangeal, radial head and scaphoid fractures had an excess in less dependent neighbourhoods. This may reflect the fact that employment contributes to this index and is a common source of injury in hand fractures. DRF was minimally affected by dependency which may reflect the fact that misfortune, bone fragility and frailty mechanisms are more important. Conversely, PHF had the highest dependency differential with almost a $20 \%$ excess in the upper double-quartile as compared to the lower. The may reflect a fragility/frailty profile where larger numbers of older retired individuals experience these fractures. This is further informed by graph of the sex-specific lowest quintile data which shows an excess preferentially in older women. The conflation of age and retirement in the dependency index, is consistent with PHF having the oldest mean age of the fracture subgroups.

The largest, and most unexpected, differences in social deprivation indices were observed with respect to residential instability which was observed as a global difference, and which was consistently manifested across most fracture subtypes. This index reflects residential home security, ownership and occupancy and is comprised of indicators of living alone, proportion of adults, number of people per dwelling, proportion who are single/divorced/widowed, and the proportion who have moved in the past 5 years. How these factors affect fracture risk is unclear and cannot be defined in this observational cohort. The potential mechanisms are many including precarious employment, higher rates of manual labour, less social support for assistance with tasks, risktaking, and environments with more fall hazards. Housing deprivation has been shown to relate to other health outcomes through complex mechanisms[34]. 
Social disadvantage can affect bone quality/strength to resist fractures due to poor nutrition during bone development, higher rates of smoking and less engagement in health promotion behaviours including bone-protective forms of exercise[35]. Social inequity is an important factor that affects health overall and that is not resolving.[36] Despite the limitations in neighbourhood social indices as an indicator of individual risk, we found substantial indication that social deprivation may affect fracture burden. This warrants more exploration of how social deprivation leads to fracture.

\section{Limitations}

Despite the large sample, this study has several limitations. Ontario is one province and may not represent other regions of Canada or other countries. However, this data represents approximately $40 \%$ of the Canadian population and is one of the largest published upper extremity fractures cohort profiles. Since administrative databases were designed for health system management and physician remuneration purposes, there are several inherent limitations to code coverage and validity. Not all fractures would present for care, and some fractures may be missed where imaging is not performed or able to detect early fracture (e.g. scaphoid fractures) or correctly interpreted/coded (e.g. Monteggia fractures). Social indicators were at the group level, not at the individual level, which limits precision.

\section{Conclusions}

The profiles of $1 \mathrm{st}$ incident fractures in patients without a prior history of upper extremity fracture as an adult is highly variable across different fracture types. Sex and lifespan greatly influence these profiles. The patterns are suggestive of risk-taking behaviours, sex and gender mechanisms, physiological differences, sex and aging mechanisms that affect bone quality, comorbid health conditions, seasonal variations/environmental exposures and social roles/exposures as combining influences on these profiles. Elicitation of mechanisms that increase risk across all of these domains and associated effective preventive strategies across all of these domains is likely to most effectively prevent secondary fractures. Variability of fracture profiles should be considered in case management.

\section{Declarations}

Ethics approval and consent to participate: as part of the arrangement between IC/ES and the Ontario Ministry of health That for the use of routinely collected health services data in research, study specific review of study protocols is waived. In light of the waiver, individual projects undergo a review process to ensure adherence with privacy protection, and adherence to IC/ES and Ontario Ministry of Health agreements about the use of the data.

Consent for publication: no individual data is presented

\section{Availability of data and materials:}

The data that support the findings of this study are available from IC/ES but restrictions apply to the availability of these data, which were used under a sharing agreement between the $1^{\text {st }}$ author and IC/ES specifically for the current study, and so are not publicly available. IC/ES share some summary data publicly and provides information on data accessibility at https://www.ices.on.ca/Data-and-Privacy/ICES-data/Working-with-ICES-Data.

\section{Competing interests: None}

Funding: Joy MacDermid was supported by a Canadian Institutes of Health Research Chair in Gender, Work and Health and the Dr James Roth Chair in Musculoskeletal Measurement and Knowledge Translation and is a Scholar in the ICES Faculty Scholars Program, London, Ontario. This work was supported by a Bone and Joint Institute Catalyst Grant and a Team Bone Grant (CIHR FRN: 122070). This study was also supported by ICES, which is funded by an annual grant from the Ontario Ministry of Health and Long-Term Care (MOHLTC).

Authors' contributions: J MacDermid contributed to the conceptualization and design of the study, analyzed and interpreted the data and drafted the manuscript. K Faber provided expert advice on upper extremity fractures and coding of surgical cases. Andrew McClure, Lucie Richard, and Susan Jaglal provided insights into health services methods, coding, ICES procedures and analysis. Andrew McClure had full access to study data and analyzed the data under the guidance of the first author. All the authors approved the final version to be published, contributed important intellectual content and agreed to act as guarantors of the work.

Acknowledgements: Surgeons at Hand and Upper Limb Centre, London Ontario (George Athwal and Graham King) provided insights into how fracture codes are used and clinical relevance, and research directions. Salimah.Shariff and Brittany Allen provided admin and training support.

This study was supported by ICES, which is funded by an annual grant from the Ontario Ministry of Health and Long-Term Care (MOHLTC). The opinions, results, and conclusions reported in this paper are those of the authors and are independent from the funding sources. No endorsement by ICES or the Ontario MOHLTC is intended or should be inferred. The study was completed at the ICES Western site, where core funding is provided by the Academic Medical Organization of Southwestern Ontario, the Schulich School of Medicine and Dentistry, Western University, and the Lawson Health Research Institute. Parts of this material are based on data and information compiled and provided by the Canadian Institute for Health Information (CIHI). However, the analyses, conclusions, opinions, and statements expressed herein are those of the author, and not necessarily those of $\mathrm{ClHI}$.

\section{References}

1. Kanis JA, Oden A, Johnell O, Jonsson B, De Laet C, Dawson A. The burden of osteoporotic fractures: A method for setting intervention thresholds. Osteoporos Int. 2001;12:417-27.

2. Bonafede M, Espindle D, Bower AG. The direct and indirect costs of long bone fractures in a working age US population. J Med Econ. 2013;16:169-78. 
3. Ahmed LA, Schirmer $Æ$ EH, Jørgensen L, Størmer $Æ E J$, Joakimsen $Æ$ RM. The gender- and age-specific 10-year and lifetime absolute fracture risk in Troms $\varnothing$, Norway. 2009;:441-8.

4. Hodsman AB, Leslie WD, Tsang JF, Gamble GD. 10-year probability of recurrent fractures following wrist and other osteoporotic fractures in a large clinical cohort: an analysis from the Manitoba Bone Density Program. Arch Intern Med. 2008;168:2261-7.

5. Klotzbuecher CM, Ross PD, Landsman PB, Abbott TA, Berger M. Patients with Prior Fractures Have an Increased Risk of Future Fractures: A Summary of the Literature and Statistical Synthesis. J Bone Miner Res. 2010;15:721-39. doi:10.1359/jbmr.2000.15.4.721.

6. Martinez-Huedo MA, Jiménez-García R, Mora-Zamorano E, Hernández-Barrera V, Villanueva-Martinez M, Lopez-De-Andres A. Trends in incidence of proximal humerus fractures, surgical procedures and outcomes among elderly hospitalized patients with and without type 2 diabetes in Spain (20012013). BMC Musculoskelet Disord. 2017.

7. Ootes D, Lambers KT, Ring DC. The epidemiology of upper extremity injuries presenting to the emergency department in the United States. Hand (N Y). 2012.

8. Karl JW, Olson PR, Rosenwasser MP. The epidemiology of upper extremity fractures in the United States, 2009. J Orthop Trauma. 2015;29:e242-4.

9. Cummings SR, Melton LJ (III). Epidemiology and outcomes of osteoporotic fractures. Lancet. 2002;359:1761-7.

10. Kennedy CC, loannidis G, Rockwood K, Thabane L, Adachi JD, Kirkland S, et al. A Frailty Index predicts 10 -year fracture risk in adults age 25 years and older: results from the Canadian Multicentre Osteoporosis Study (CaMos). Osteoporos Int. 2014;25:2825-32.

11. Calvo E, Morcillo D, Foruria AM, Redondo-Santamaría E, Osorio-Picorne F, Caeiro JR. Nondisplaced proximal humeral fractures: High incidence among outpatient-treated osteoporotic fractures and severe impact on upper extremity function and patient subjective health perception. J Shoulder Elb Surg. 2011;20:795-801.

12. Schuit SCE, Van Der Klift M, Weel AEAM, De Laet CEDH, Burger H, Seeman E, et al. Fracture incidence and association with bone mineral density in elderly men and women: The Rotterdam Study. Bone. 2004;34:195-202.

13. Baron J, Karagas M, Barrett J, Kniffin W. Basic Epidemiology of Fractures of the Upper and Lower Limb among American over 65 Years of Age. Epidemiology. 1996;7:612-8.

14. Palvanen M, Kannus P, Niemi S, Parkkari J. Update in the epidemiology of proximal humeral fractures. Clin Orthop Relat Res. 2006 ;:87-92.

15. Karl P. R.;Rosenwasser, M. P. JW. O. The Epidemiology of Upper Extremity Fractures in the United States, 2009. J Orthop Trauma. 2015;29:e242-4.

16. Taylor CA, Braza D, Rice JB, Dillingham T. The Incidence of Peripheral Nerve Injury in Extremity Trauma. Am J Phys Med Rehabil. 2008;87:381-5. doi:10.1097/PHM.0b013e31815e6370.

17. Hux JE, Ivis F, Flintoft V, Bica A. Diabetes in Ontario: Determination of prevalence and incidence using a validated administrative data algorithm. Diabetes Care. 2002;25:512-6.

18. Widdifield J, Bernatsky S, Paterson JM, Tu K, Ng R, Thorne JC, et al. Accuracy of Canadian health administrative databases in identifying patients with rheumatoid arthritis: A validation study using the medical records of rheumatologists. Arthritis Care Res. 2013;65:1582-91.

19. Widdifield J, Bombardier C, Bernatsky S, Paterson JM, Green D, Young J, et al. An administrative data validation study of the accuracy of algorithms for identifying rheumatoid arthritis: The influence of the reference standard on algorithm performance. BMC Musculoskelet Disord. $2014 ; 15$.

20. Allin S, Munce S, Jaglal S, Butt D, Young J, Tu K. Capture of osteoporosis and fracture information in an electronic medical record database from primary care. 2014

21. Charlson M, Szatrowski TP, Peterson ' J, Ggld J. Validation of A Combined Comorbidity Index. J Clin Epidemiol. 1994;47:1245-51.

22. D;Hoore W, Bouckaert A, Tilquin C. Practical considerations on the use of the Charlson Comorbidity Index with administrative databases. J Clin Epidemiol. 1996;49:1429-33.

23. Yurkovich M, Avina-Zubieta JA, Thomas J, Gorenchtein M, Lacaille D. A systematic review identifies valid comorbidity indices derived from administrative health data. J Clin Epidemiol. 2015;68:3-14.

24. Matheson FI, Moloney G, van Ingen T. 2016 Ontario Marginalization Index. Toronto, ON: Providence St. Joseph's and St. Michael's Healthcare; 2018.

25. Moin JS, Moineddin R, Upshur REG. Measuring the association between marginalization and multimorbidity in Ontario, Canada: A cross-sectional study. J Comorbidity. 2018;8:2235042X1881493.

26. Statistics Canada. Age (in Single Years) and Average Age and Sex for the Population of Canada and Forward Sortation Areas, 2016 Census - $100 \%$ Data. https://www12.statcan.gc.ca/census-recensement/2016/dp-pd/dt-td/Rp-eng.cfm?

LANG $=E \& A P A T H=3 \& D E T A I L=0 \& D I M=0 \& F L=A \& F R E E=0 \& G C=0 \& G I D=0 \& G K=0 \& G R P=1 \& P I D=109790 \& P R I D=10 \& P T Y P E=109445 \& S=0 \& S H O W A L L=0 \& S U B=($ Accessed 12 Jul 2019.

27. Seeman E. Bone quality: The material and structural basis of bone strength. J Bone Miner Metab. 2008;26:1-8.

28. Philip SS, Macdermid JC, Nair S, Walton D, Grewal R. What Factors Contribute to Falls-Related Distal Radius Fracture? J Aging Phys Act. $2019 ; 27: 392-7$.

29. Widdifield J, Paterson JM, Bernatsky S, Tu K, Tomlinson G, Kuriya B, et al. The epidemiology of rheumatoid arthritis in Ontario, Canada. Arthritis Rheumatol. 2014.

30. Lipscombe LL, Jamal SA, Booth GL, Hawker GA. The Risk of Hip Fractures in Older Individuals With Diabetes. Diabetes Care. 2007;30:835 LP - 841. doi:10.2337/dc06-1851.

31. Bonds DE, Larson JC, Schwartz A V, Strotmeyer ES, Robbins J, Rodriguez BL, et al. Risk of Fracture in Women with Type 2 Diabetes: the Women's Health Initiative Observational Study. J Clin Endocrinol Metab. 2006;91:3404-10. doi:10.1210/jc.2006-0614. 
32. Coley RL. Children's Socialization Experiences and Functioning in Single-Mother Households: The Importance of Fathers and Other Men. Child Dev. 1998;69:219-30. doi:10.1111/j.1467-8624.1998.tb06144.x.

33. Sterling RS. Gender and Race/Ethnicity Differences in Hip Fracture Incidence, Morbidity, Mortality, and Function. Clin Orthop Relat Res. 2011;469:1913-8. doi:10.1007/s11999-010-1736-3.

34. Wan C, Su S. Neighborhood housing deprivation and public health: Theoretical linkage, empirical evidence, and implications for urban planning. Habitat Int. 2016;57:11-23.

35. Algren MH, Bak CK, Berg-Beckhoff G, Andersen PT. Health-Risk Behaviour in Deprived Neighbourhoods Compared with Non-Deprived Neighbourhoods: A Systematic Literature Review of Quantitative Observational Studies. PLoS One. 2015;10:e0139297. https://doi.org/10.1371/journal.pone.0139297.

36. Newton JN, Briggs ADM, Murray CJL, Dicker D, Foreman KJ, Wang H, et al. Changes in health in England, with analysis by English regions and areas of deprivation, 1990-2013: A systematic analysis for the Global Burden of Disease Study 2013. Lancet. 2015;386:2257-74.

\section{Figures}

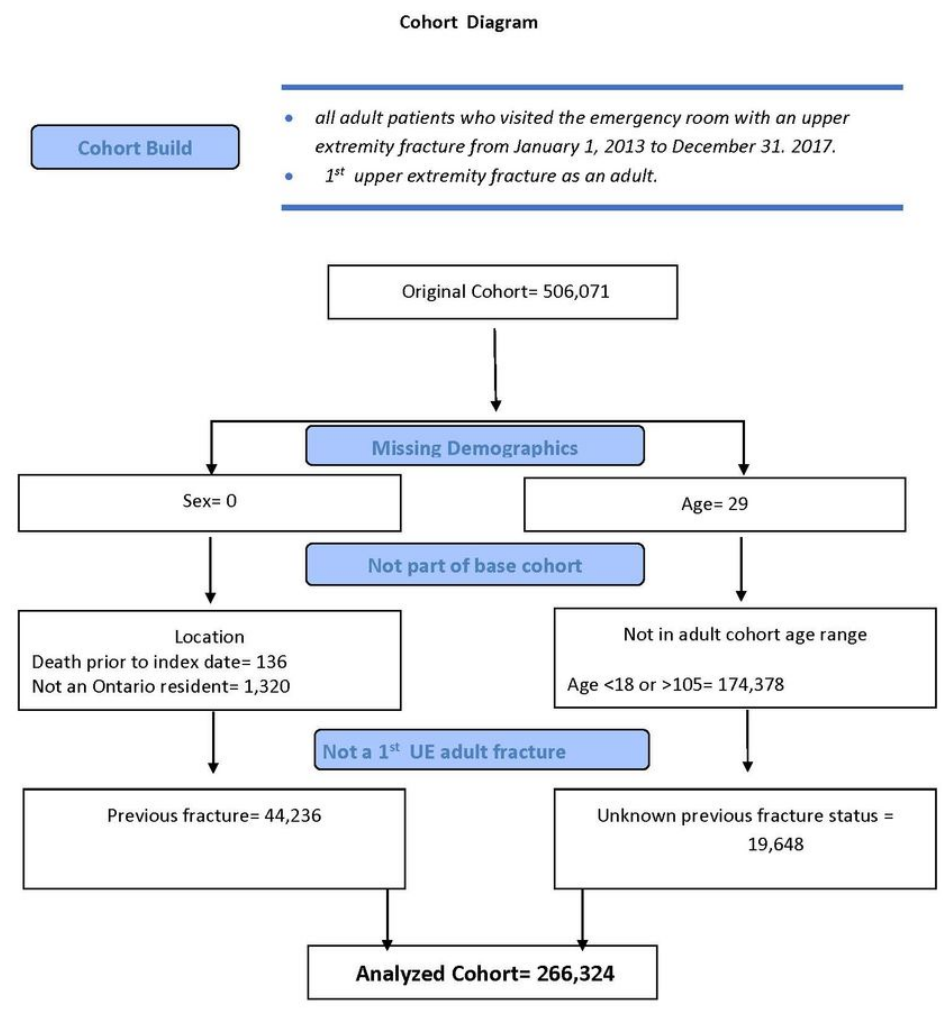

Figure 1

Cohort Diagram 
Figure 2. Number of First UE Fracture for adults by region

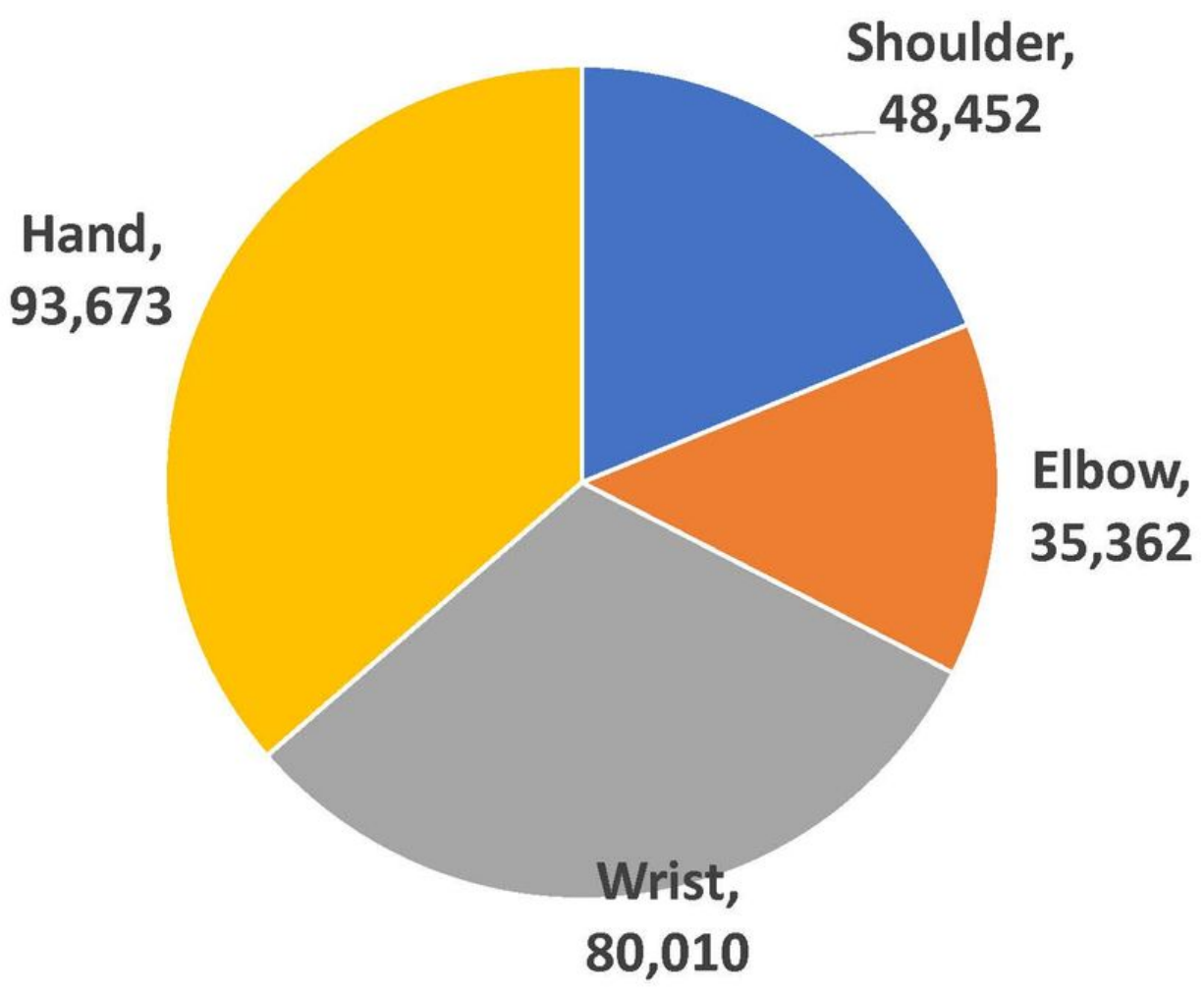

Figure 2

Number of First UE Fracture for adults by region 


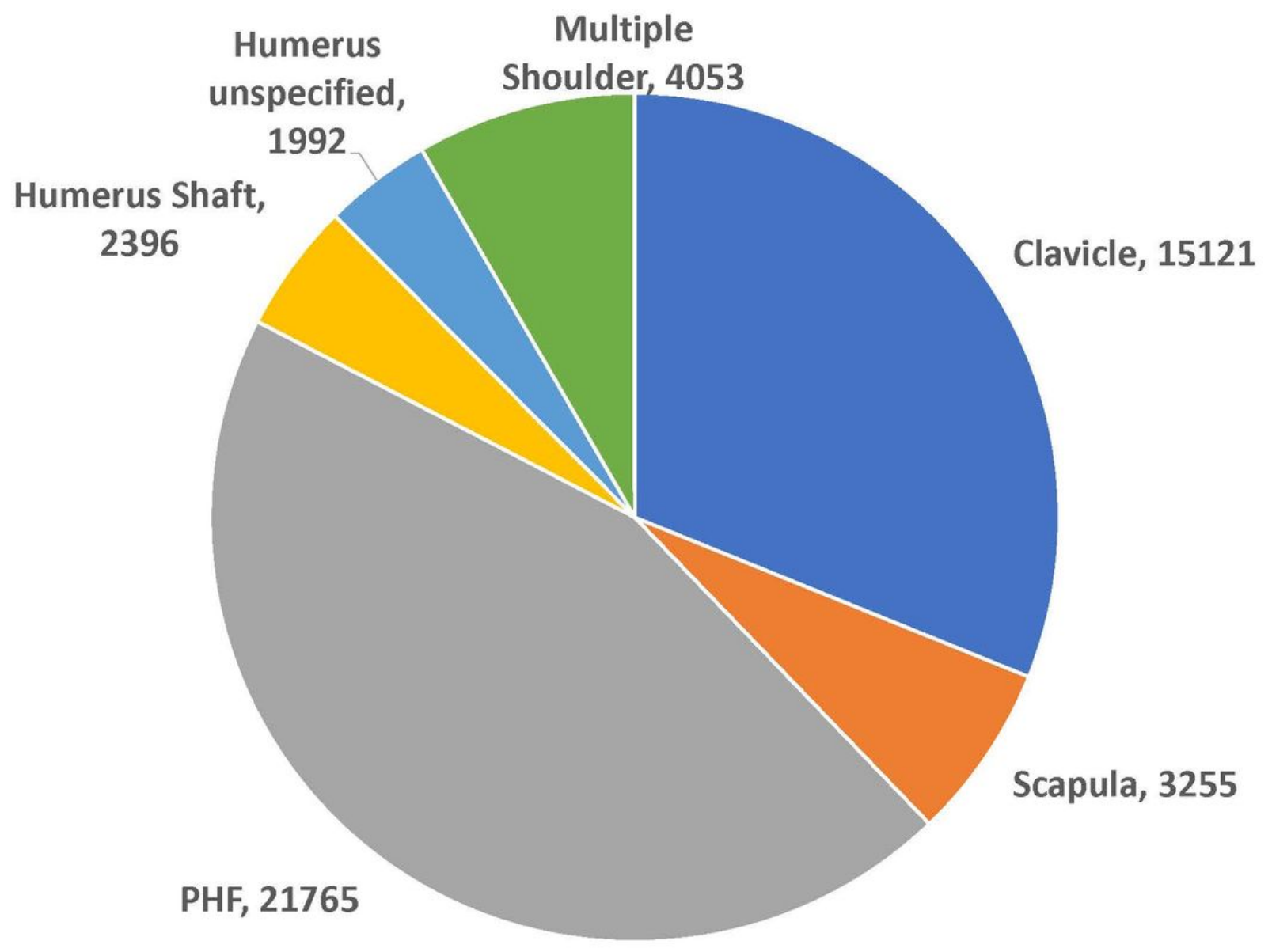

Figure 3. Distribution of Shoulder Fractures

Figure 3

Distribution of Shoulder Fractures 


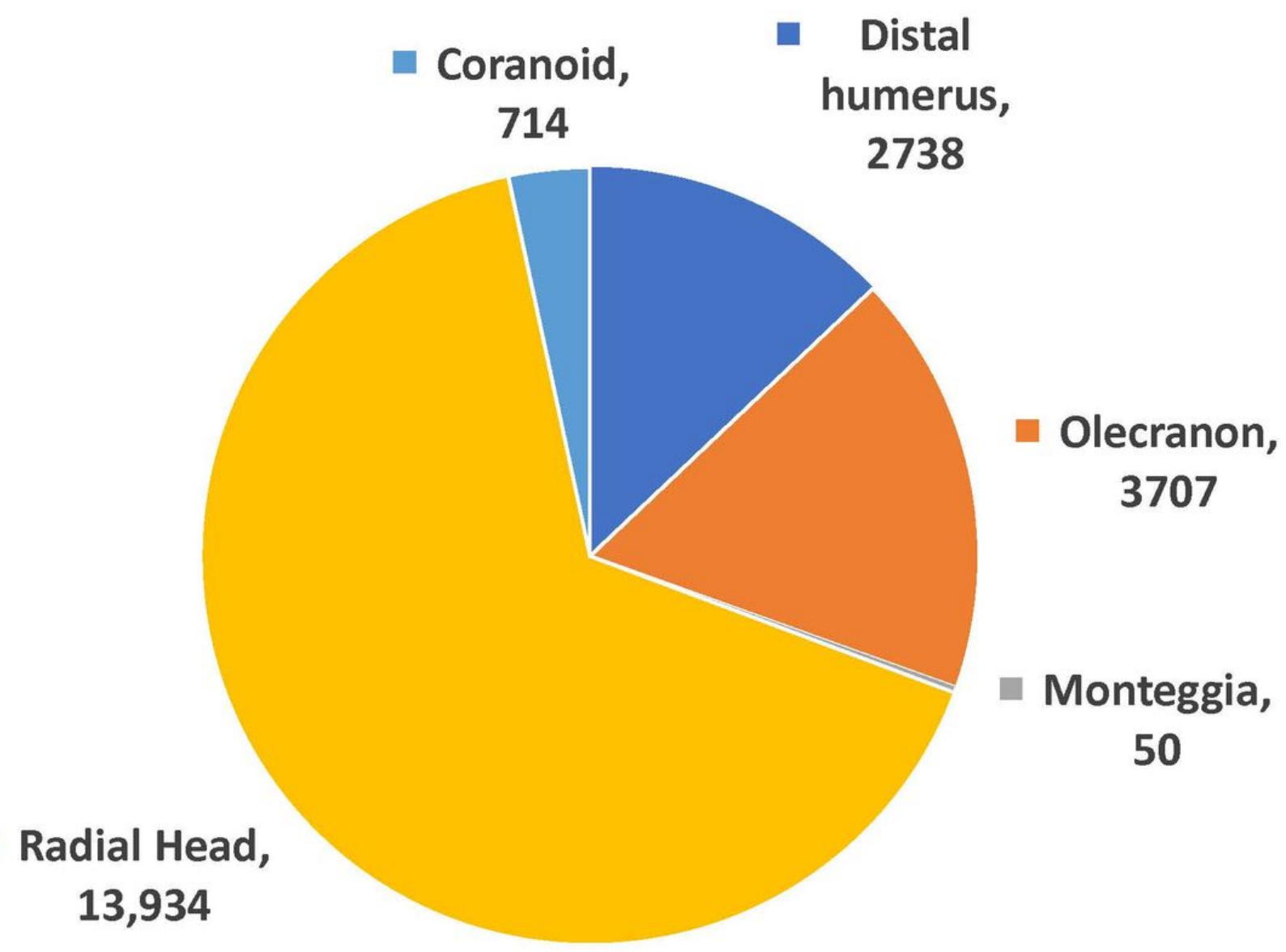

Figure 4. Distribution of Elbow Fractures

Figure 4

Distribution of Elbow Fractures 


\section{Figure 5. Distribution of Forearm and Wrist Fractures}

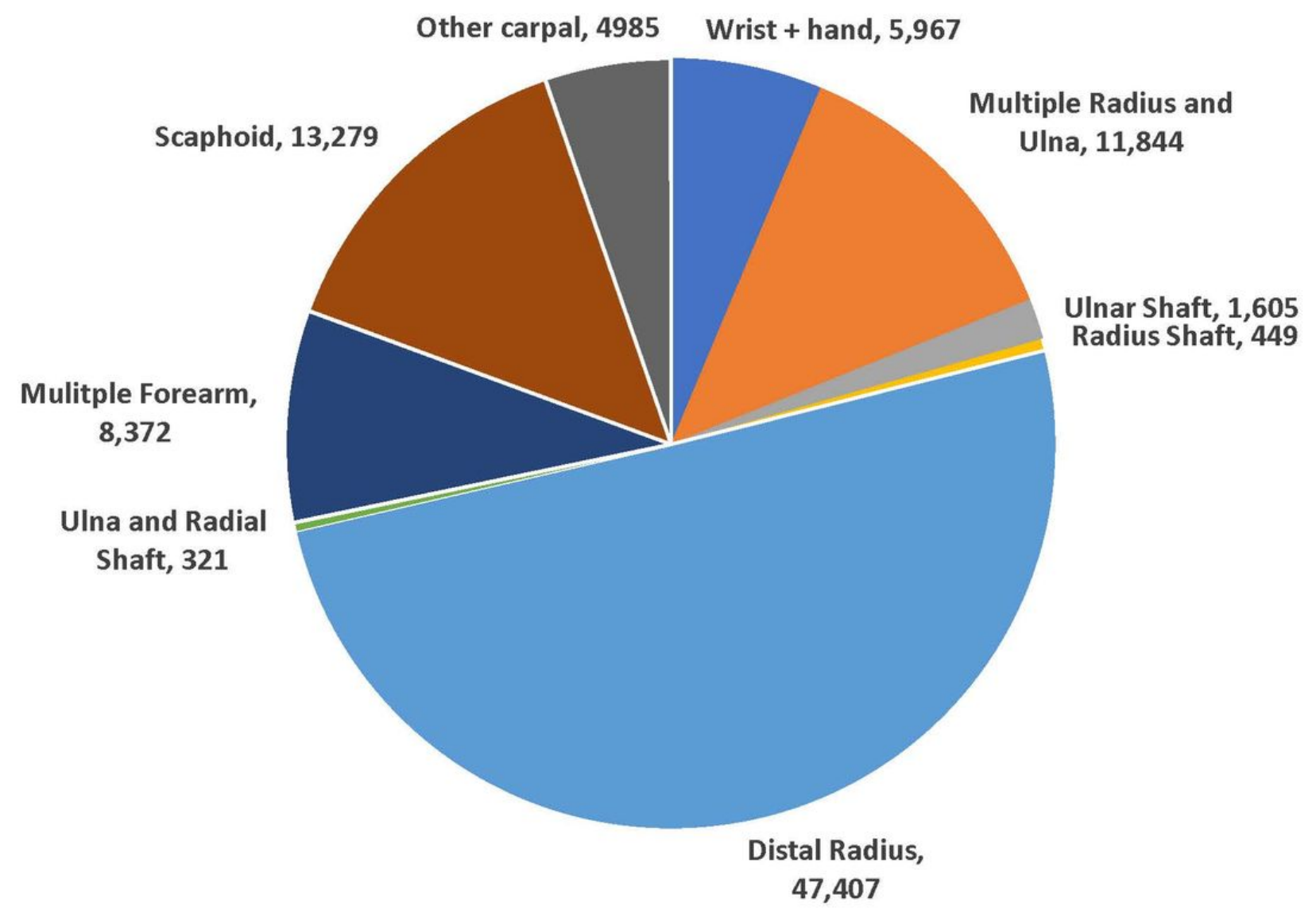

Figure 5

Distribution of Elbow Fractures 


\section{Figure 6. Distribution of Hand Fractures}

Multiple Finger, 4655 D1 MC, 2,071

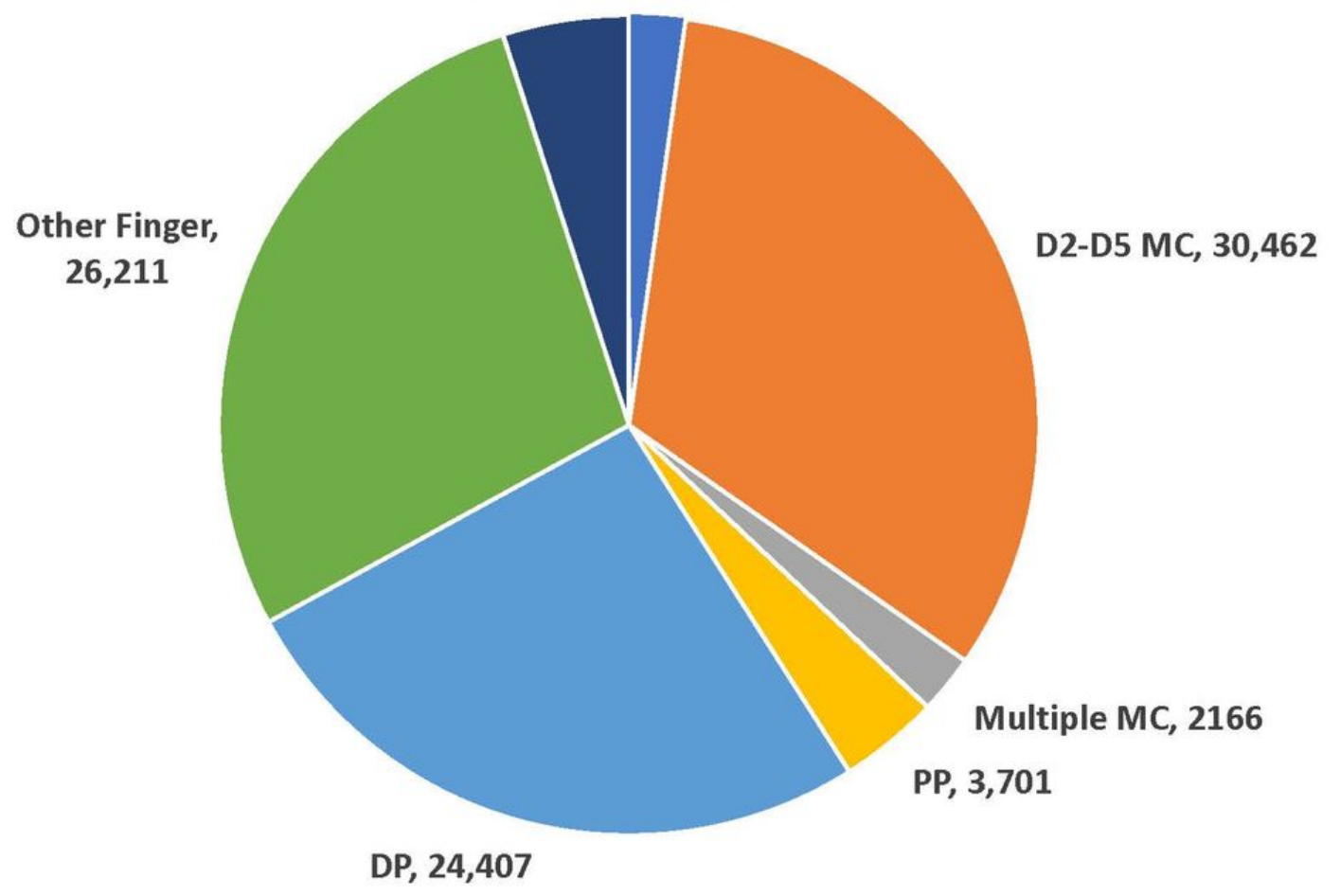

Figure 6

Distribution of Hand Fractures 
Figure 7. Major first UE fractures in men and women

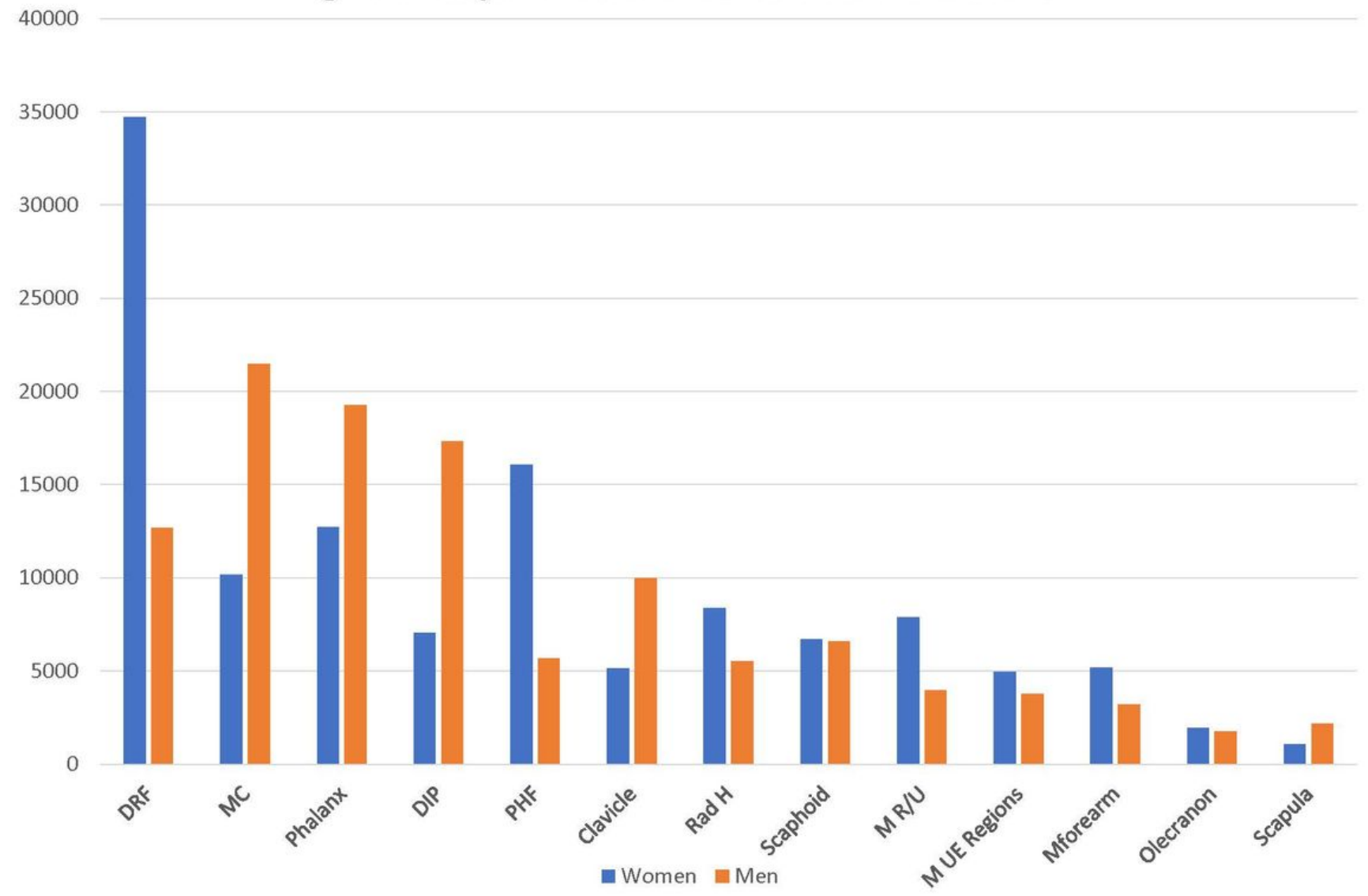

Figure 7

Major first UE fractures in men and women 
Figure 8. Major Fractures by Age Group

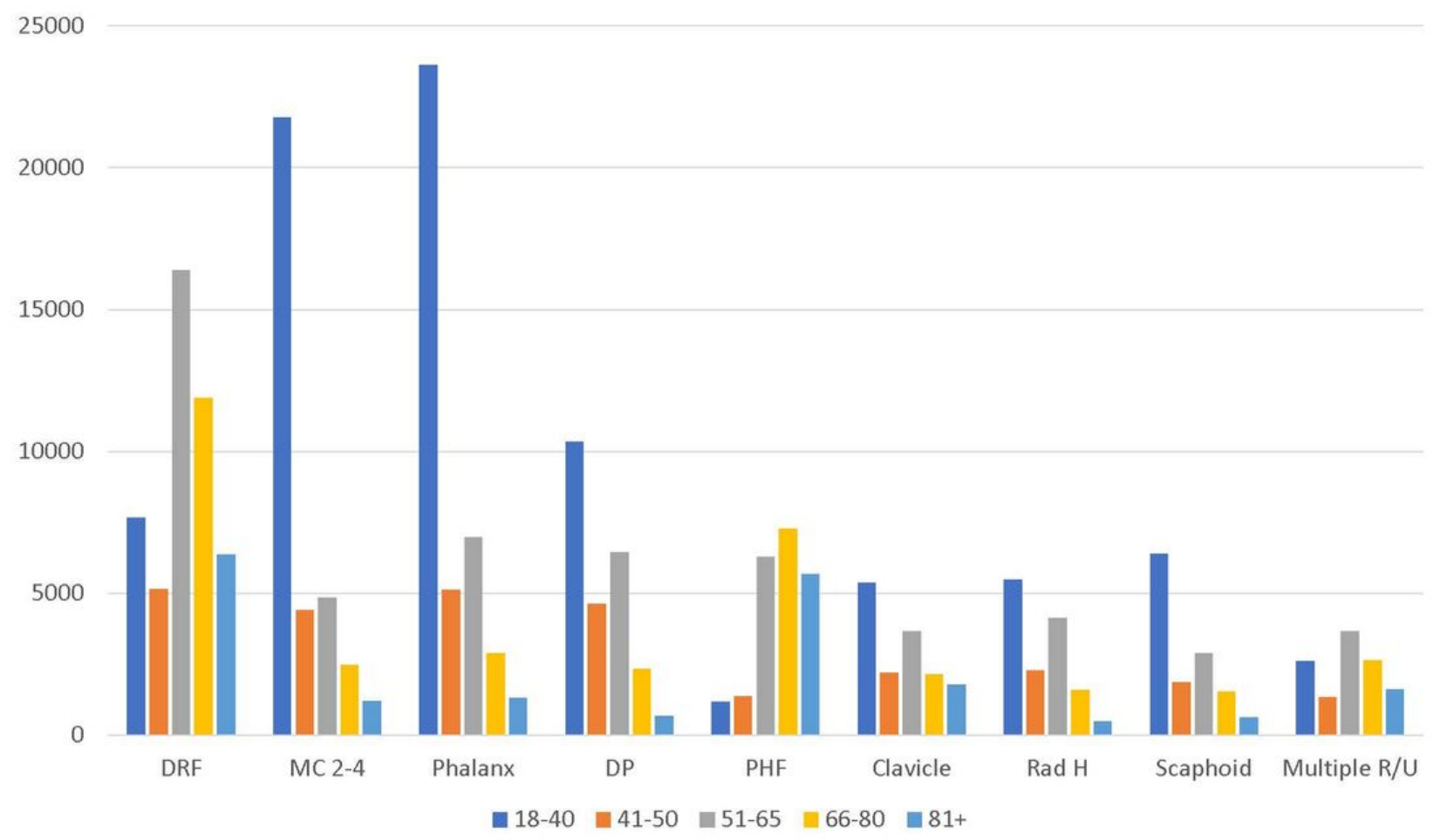

Figure 8

Major Fractures by Age Group 
Figure 9. Scapula Fractures

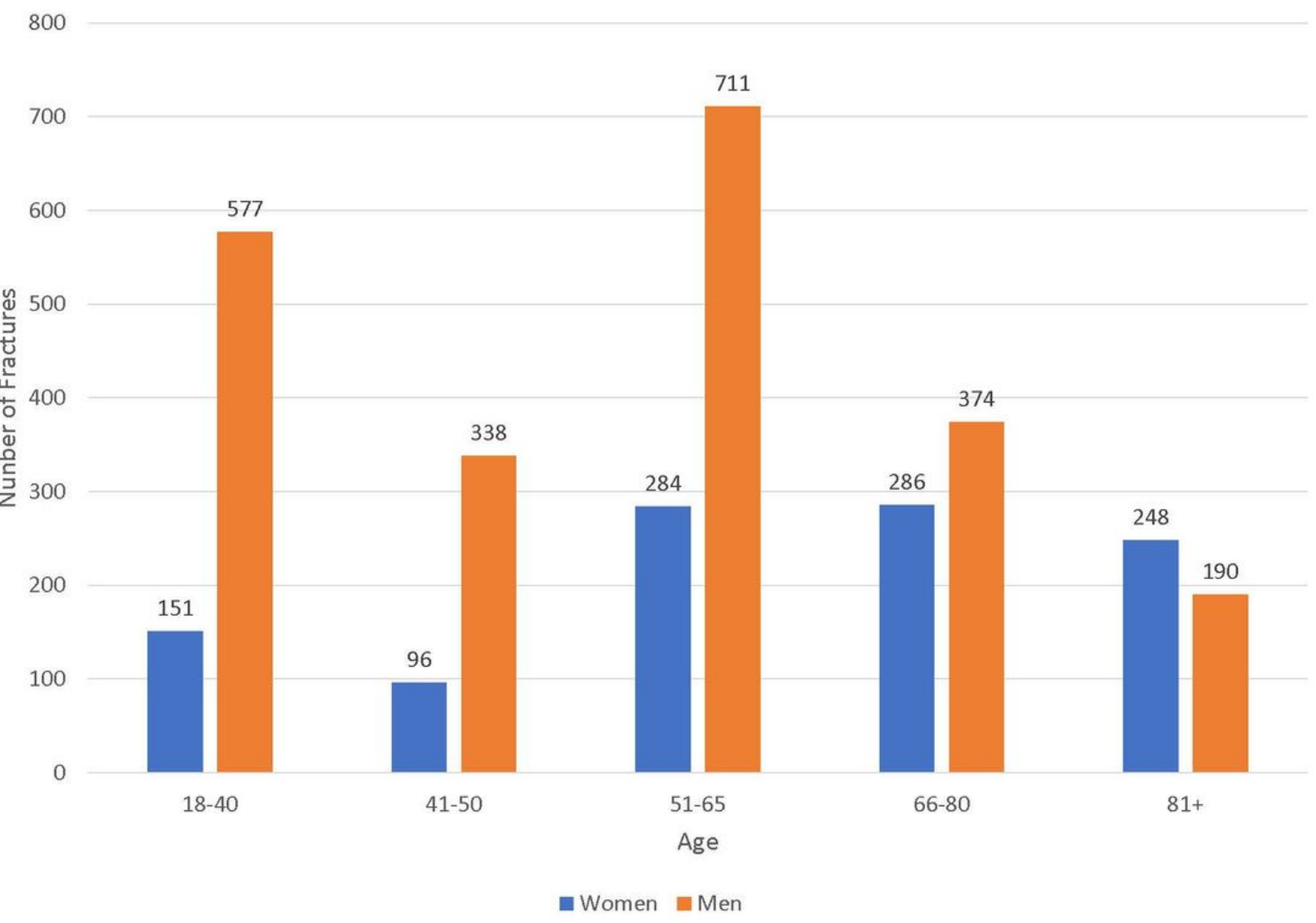

Figure 9

Scapula Fractures 
Figure 10. Clavicle Fractures

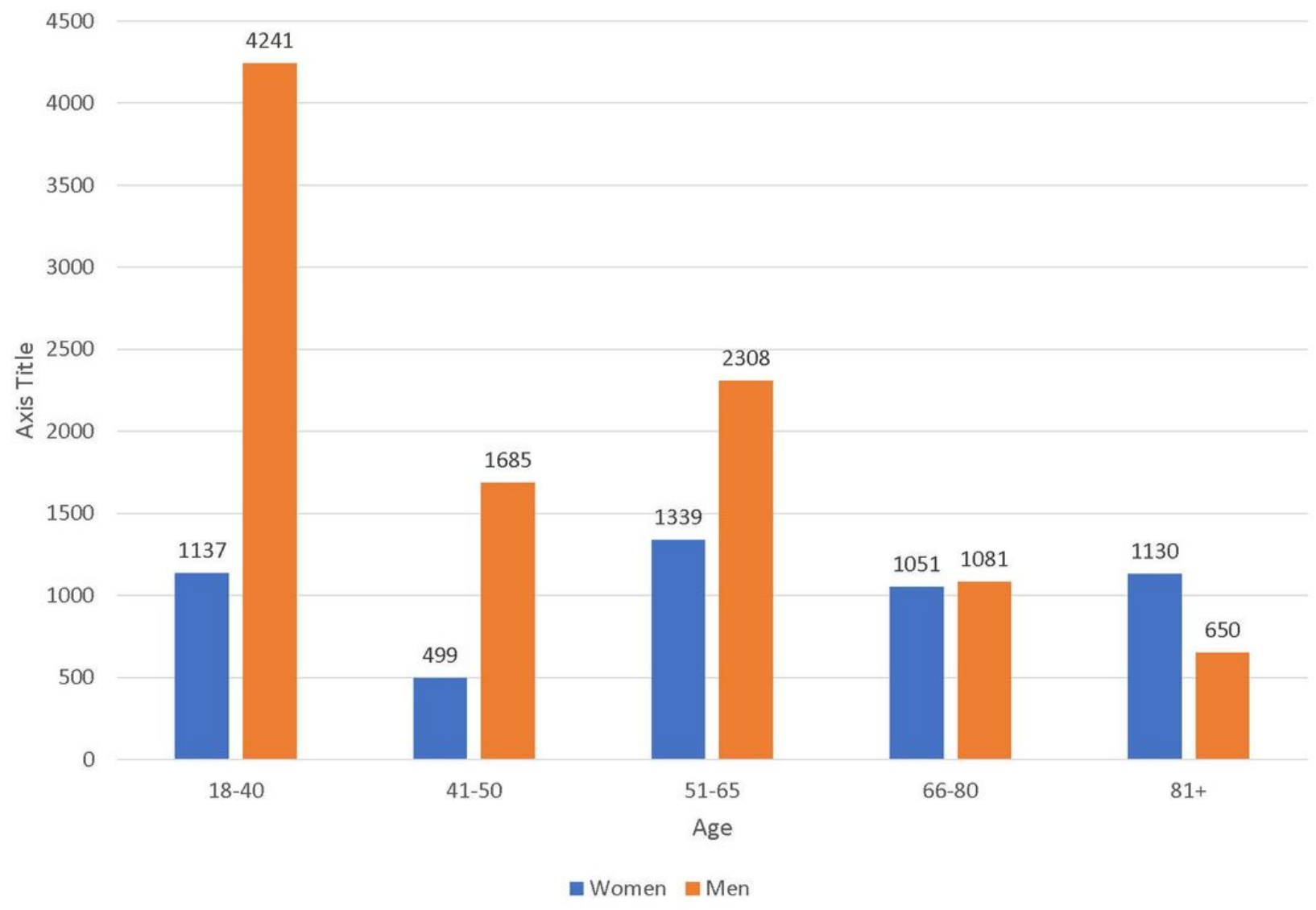

Figure 10

Clavicle Fractures 
Figure 11. Proximal Humerus Fractures

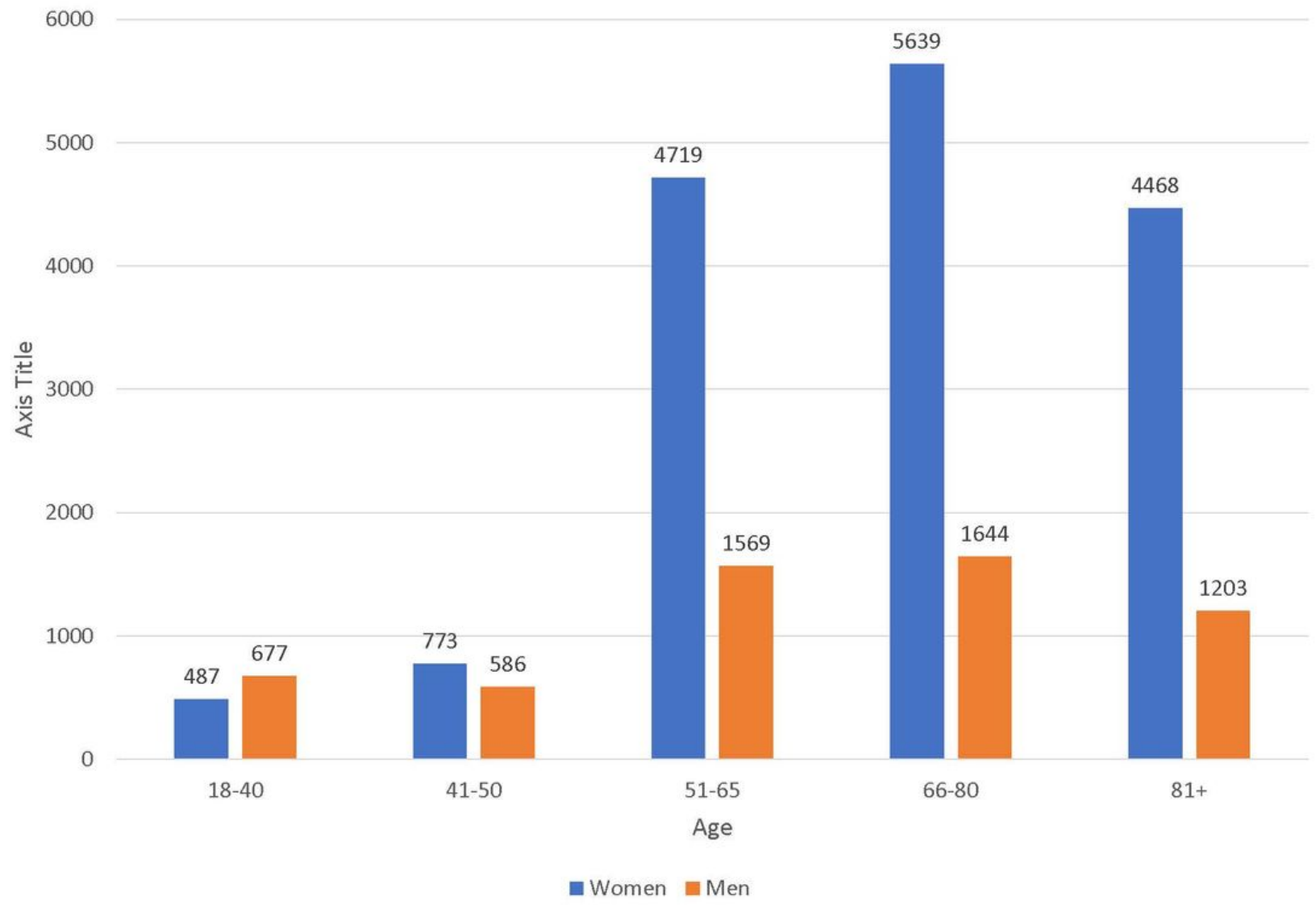

Figure 11

Proximal HumerusFractures 
Figure 12. Humerus Shaft Fractures

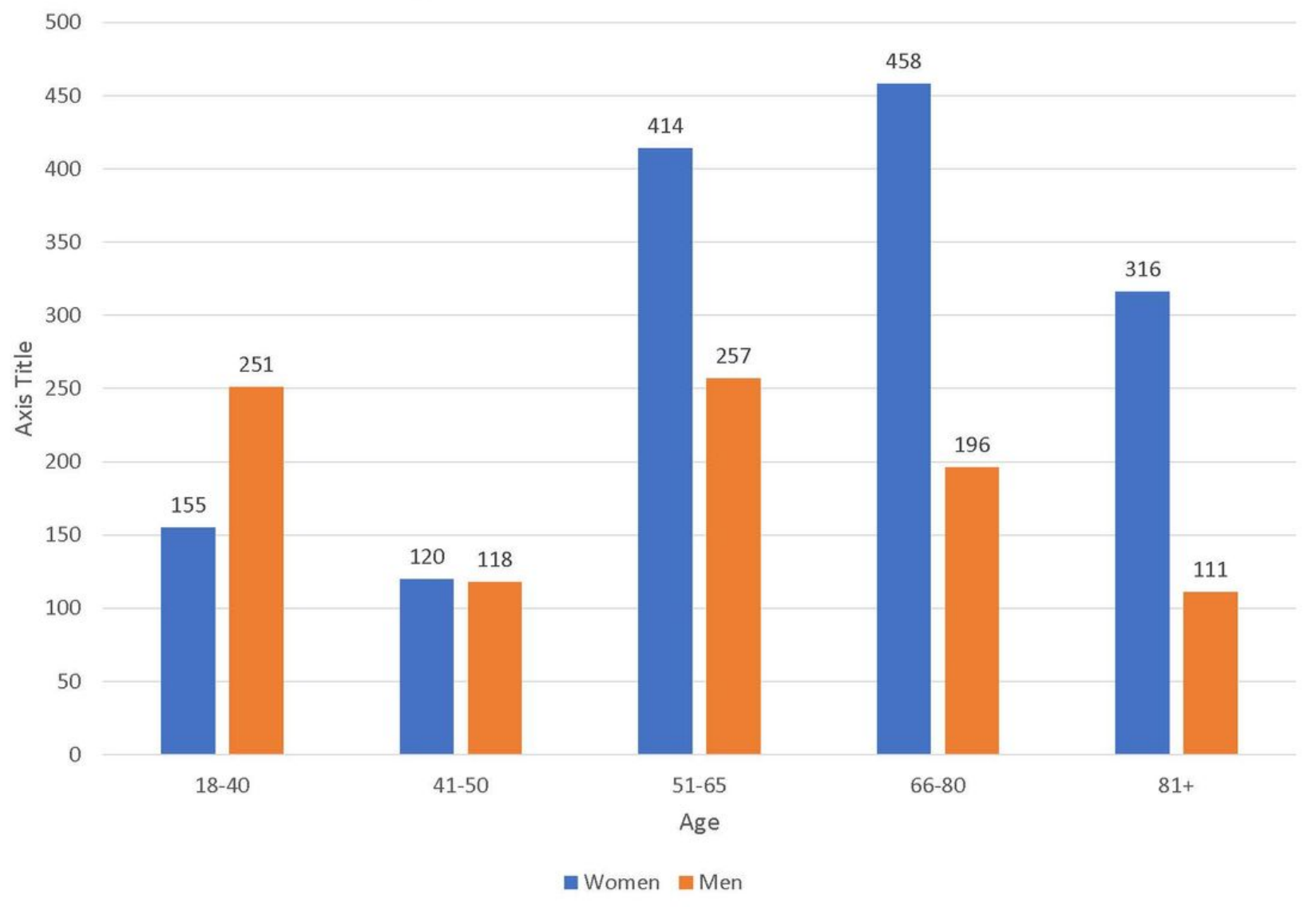

Figure 12

HumerusShaft Fractures 
Figure 13. Radial Head Fractures

3500

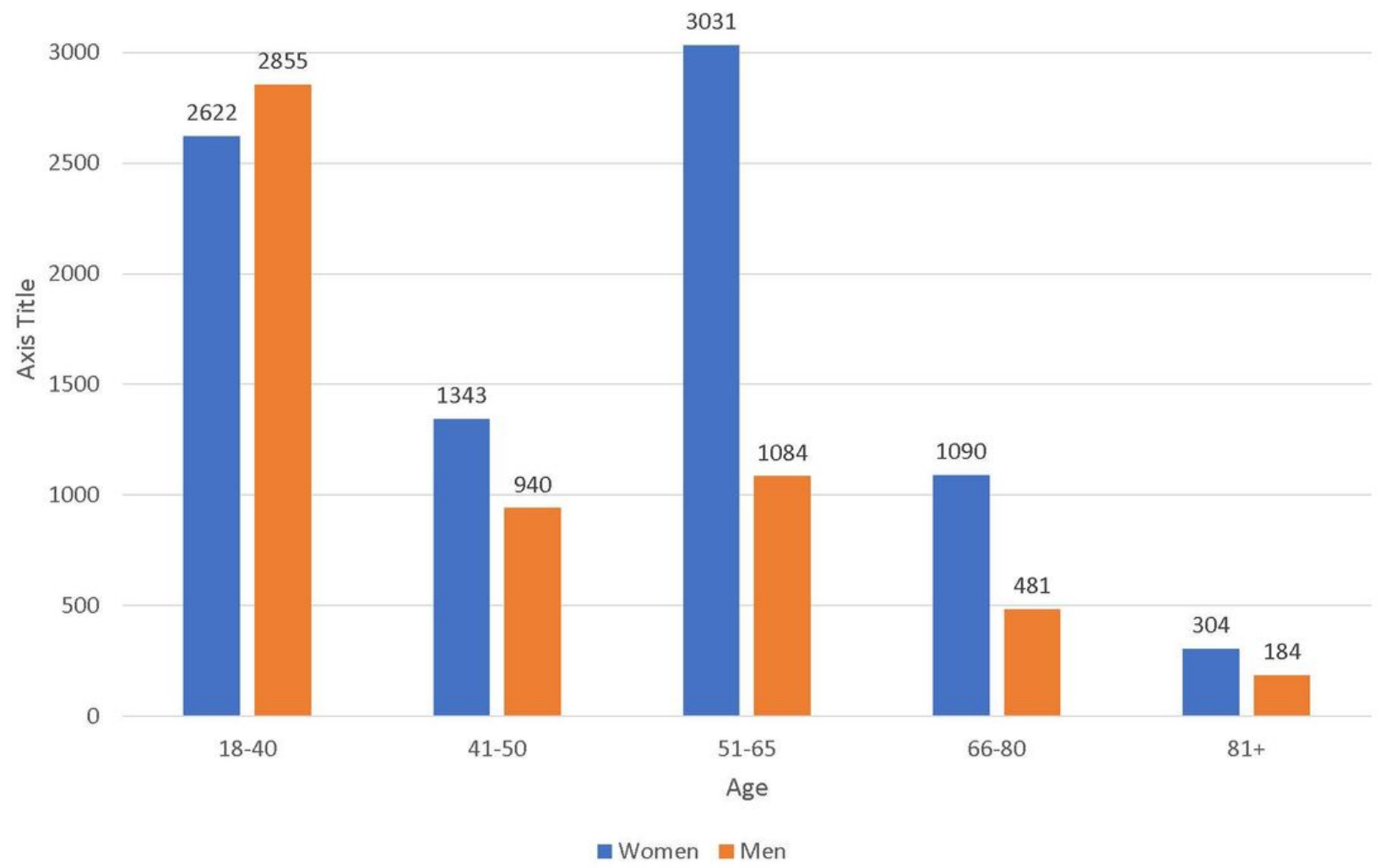

Figure 13

Radial Head Fractures 
Figure 14. Olecranon Fractures

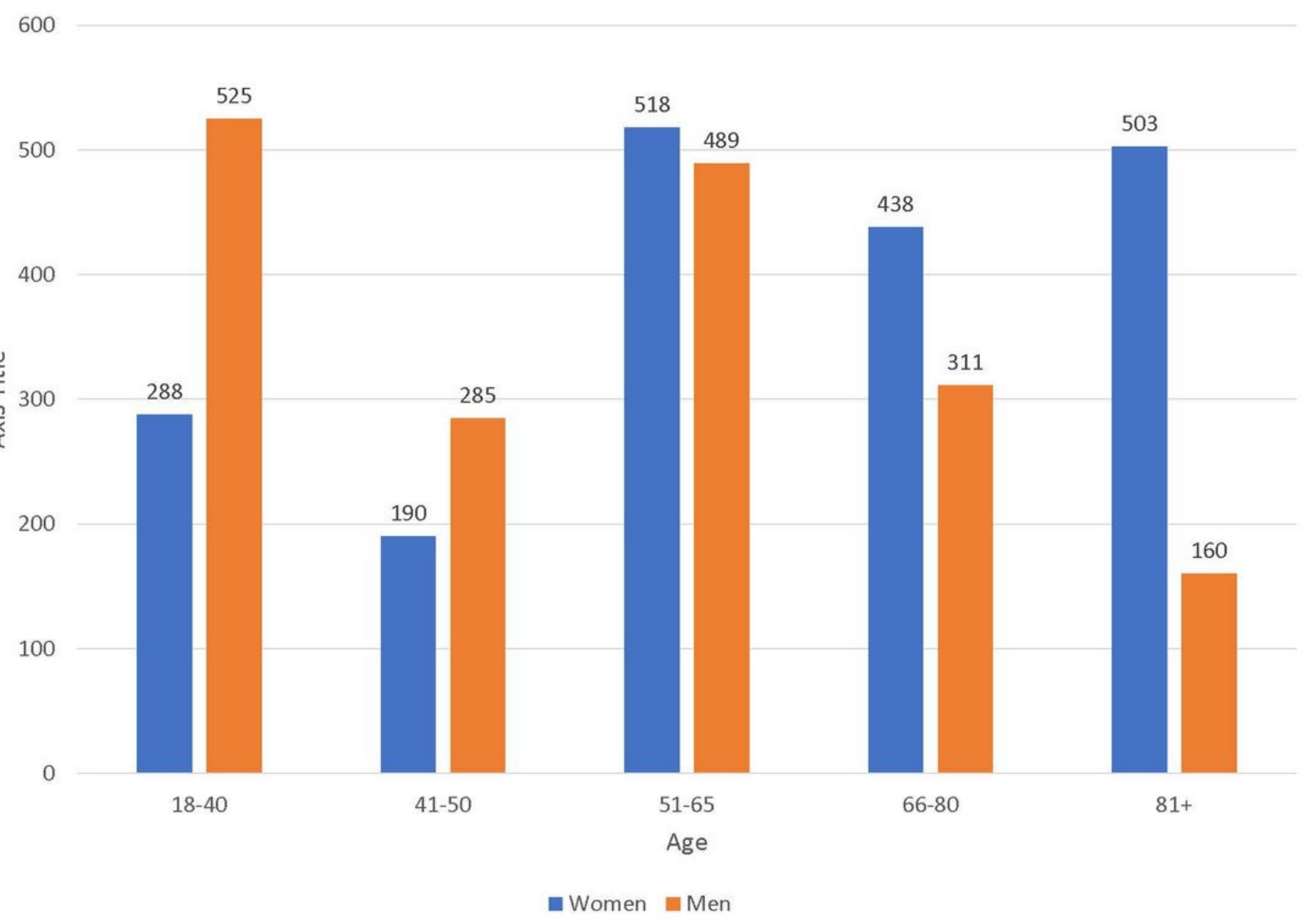

Figure 14

Olecranon Fractures 
Figure 15. Distal radius fractures

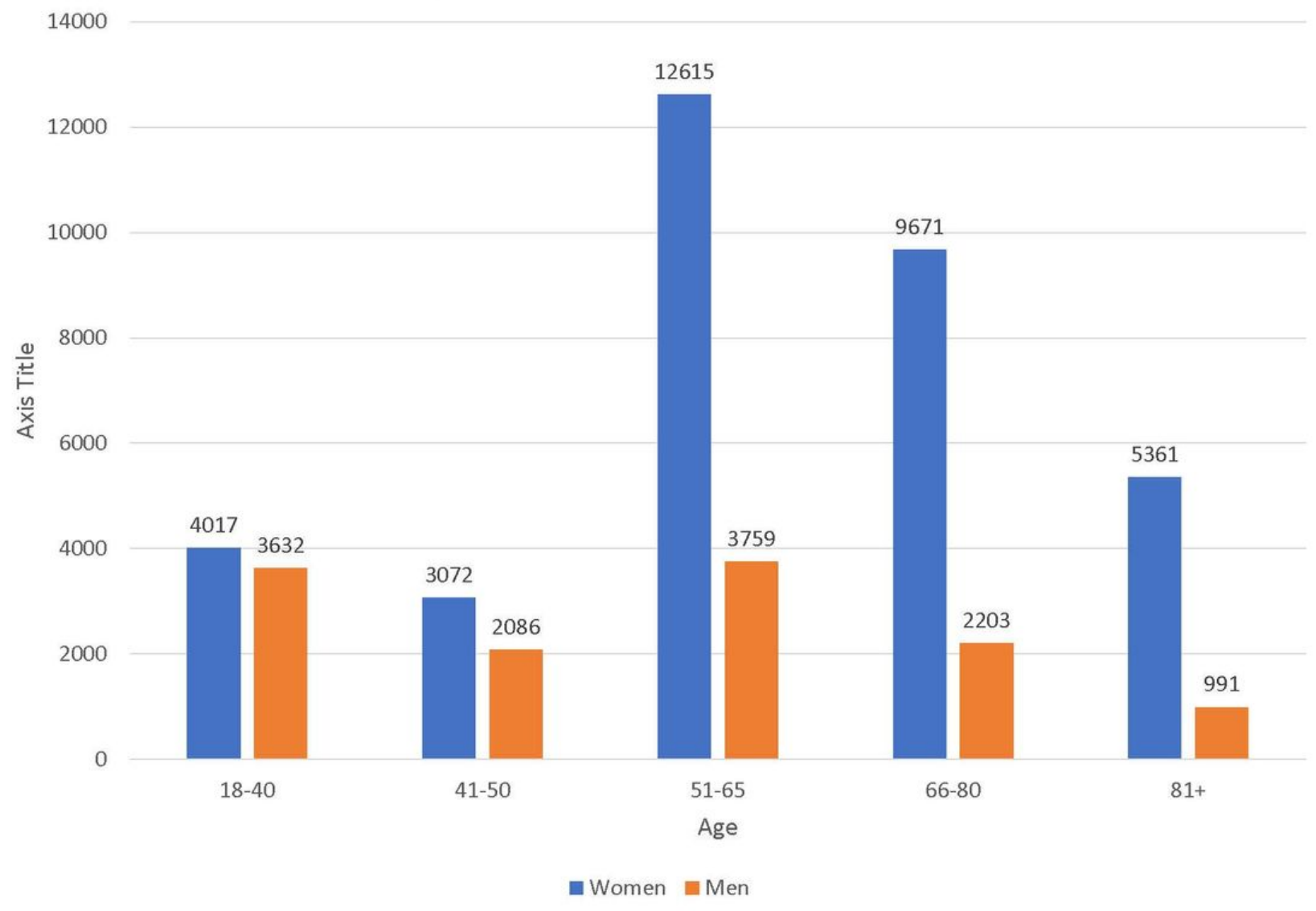

Figure 15

Distal radius fractures 
Figure 16. Scaphoid Fractures

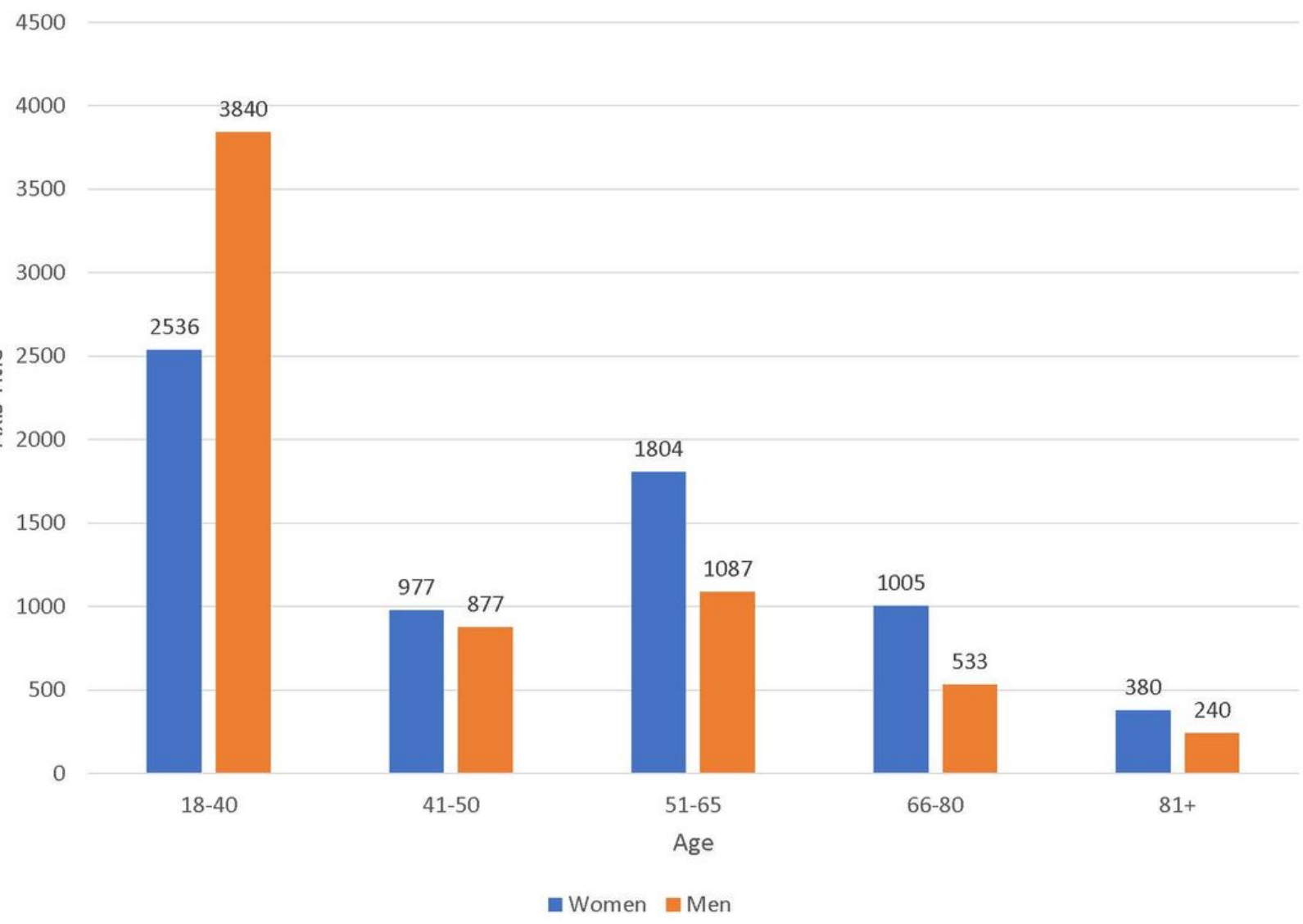

Figure 16

Scaphoid Fractures 
Figure 17. D2-D5 Metacarpal Fractures

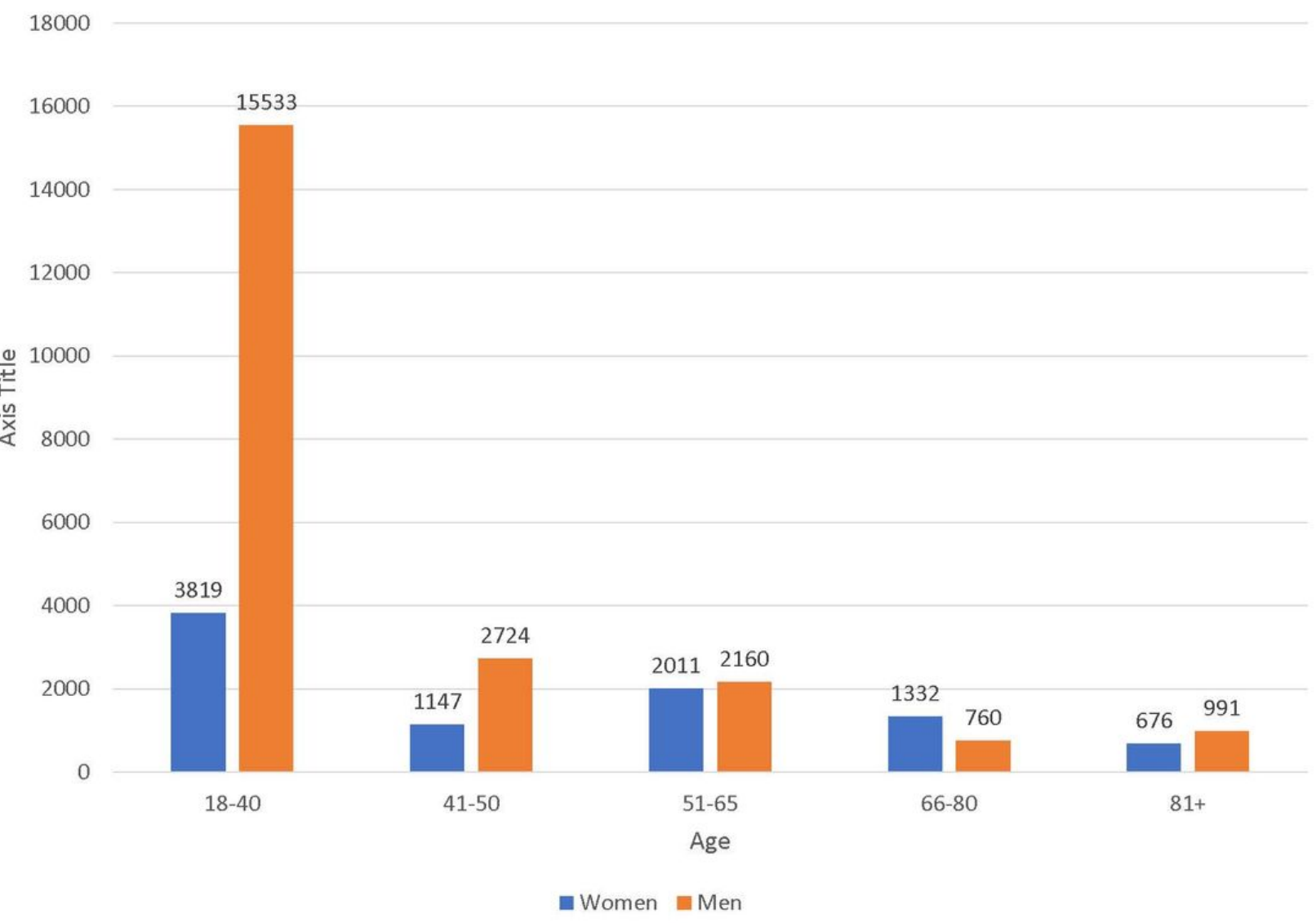

Figure 17

D2-D5 Metacarpal Fractures 
Figure 18. Proximal or Other Phalanx Fracture

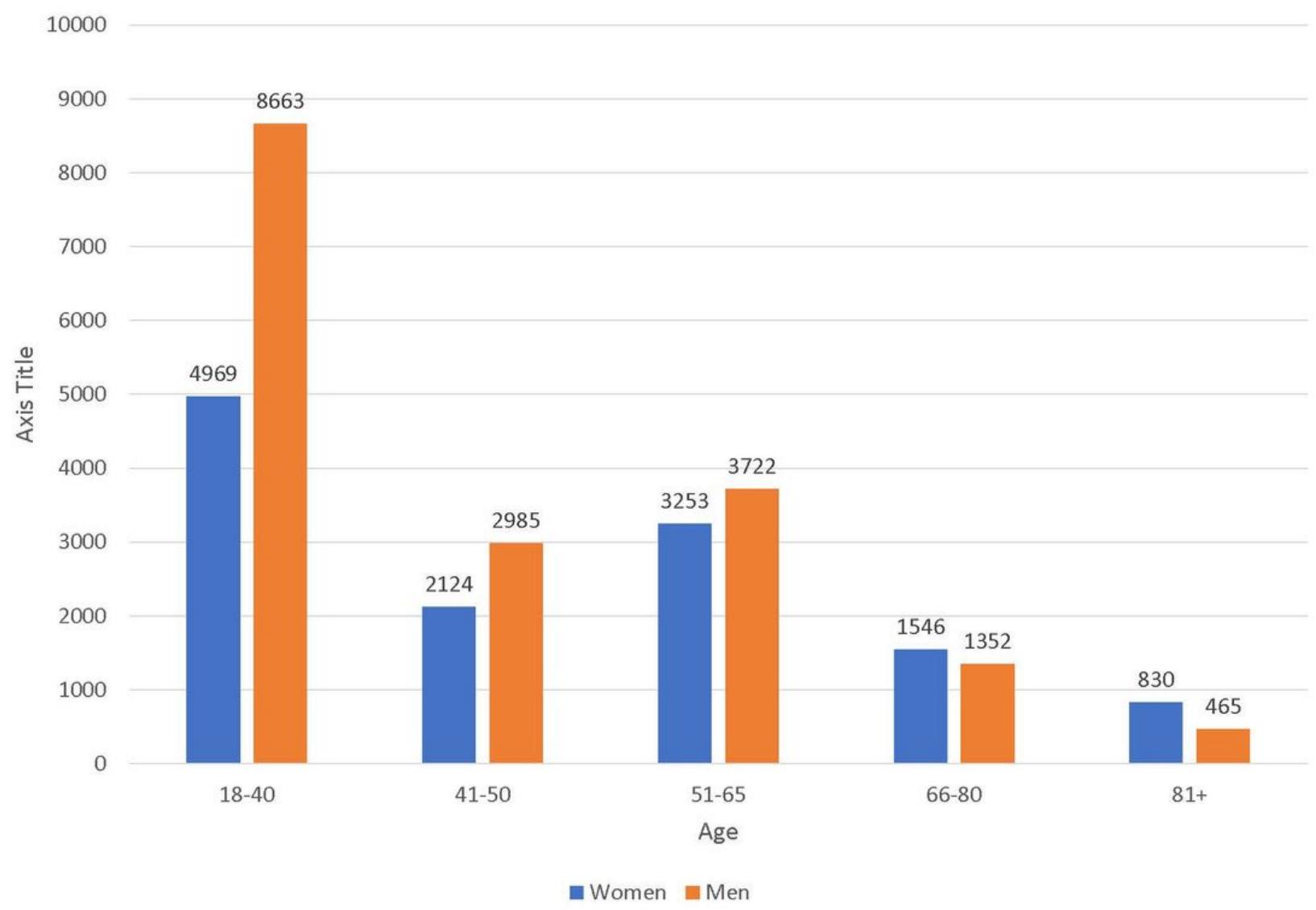

Figure 18

Proximal or Other Phalanx Fracture 
Figure 19. Distal Phalanx Fracture

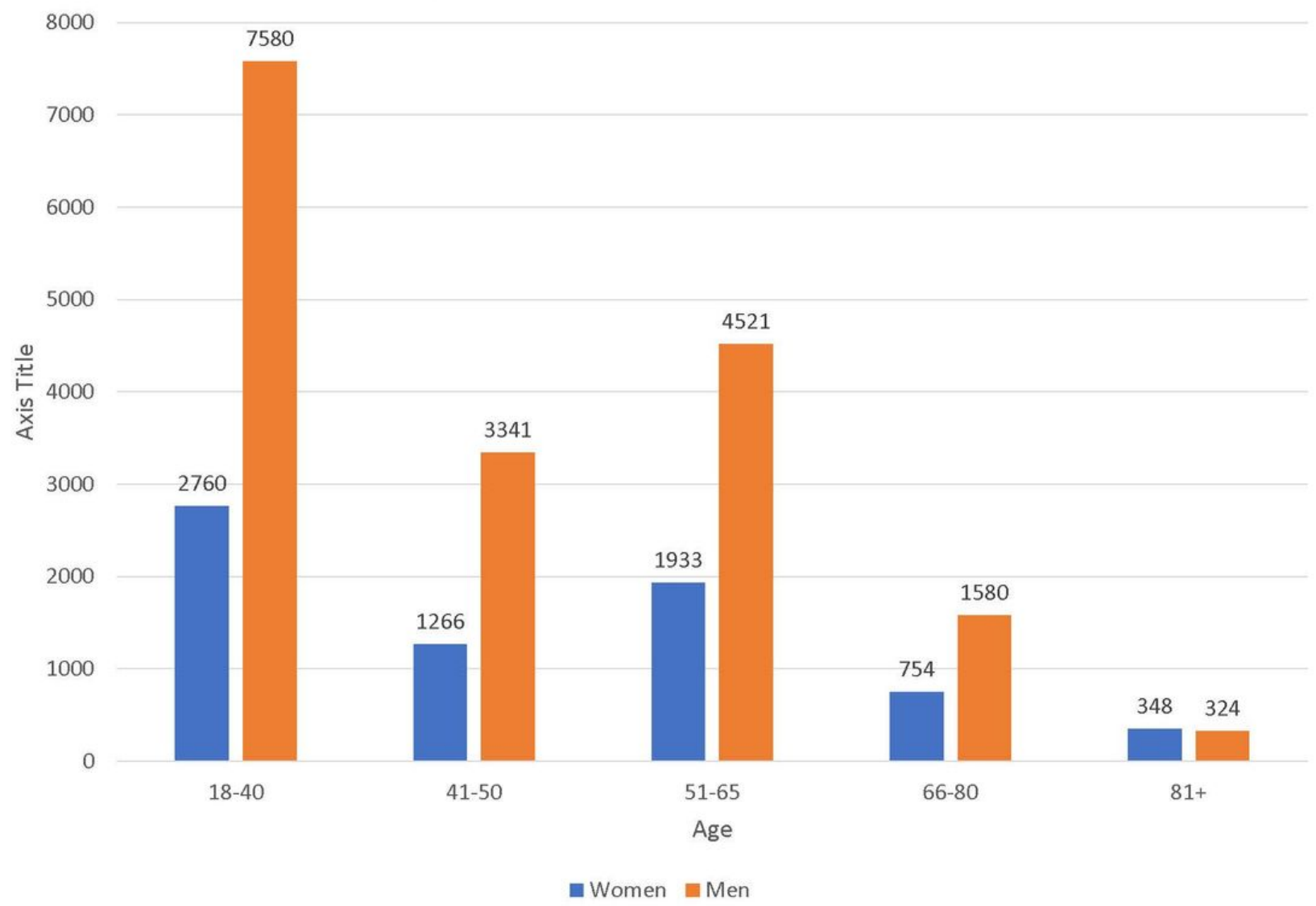

Figure 19

Distal Phalanx Fracture 
Figure 20. Multiple Regions of UE Fractures

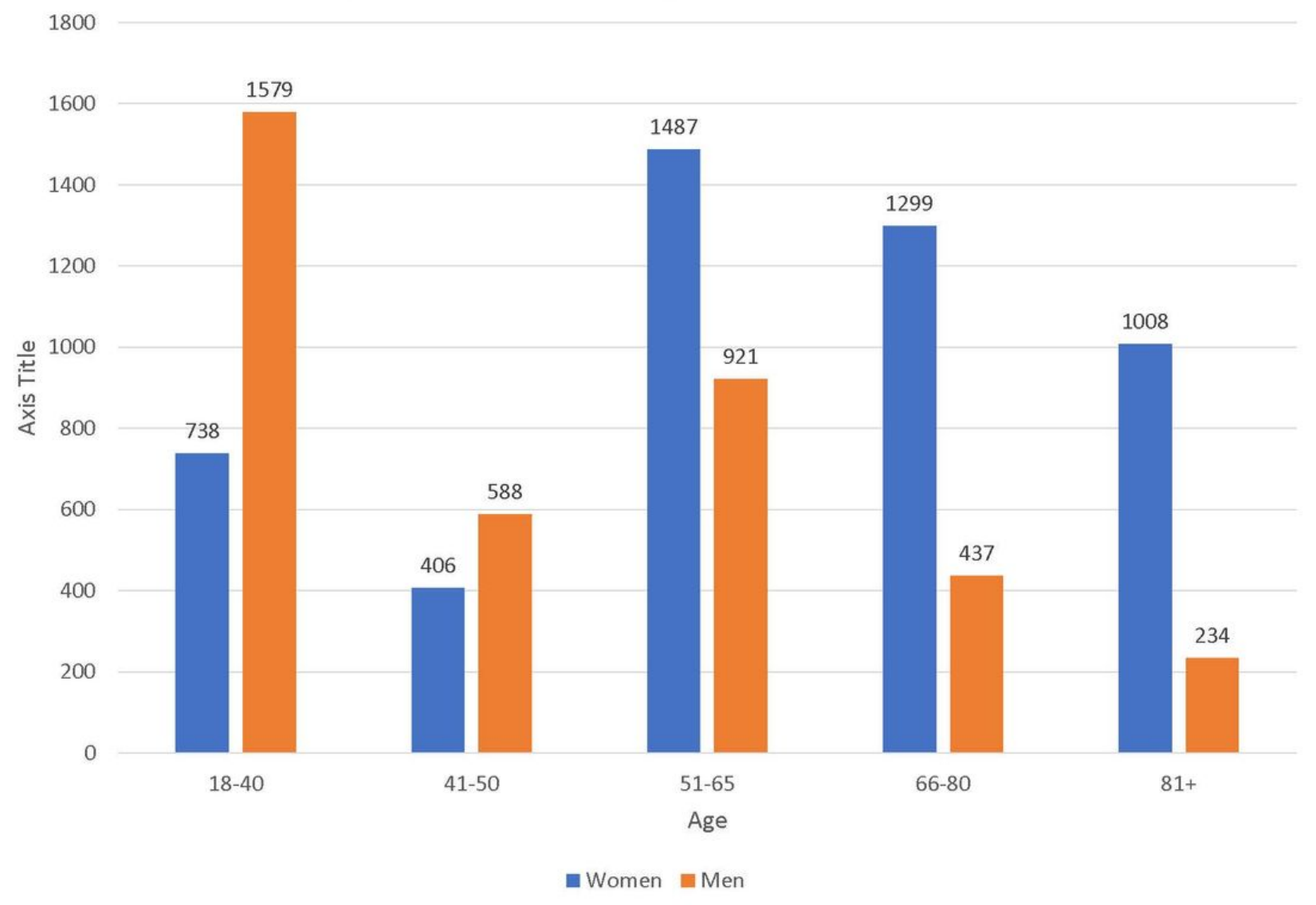

Figure 20

Multiple Regions of UE Fractures 
Figure 21. Multiple Shoulder Fractures

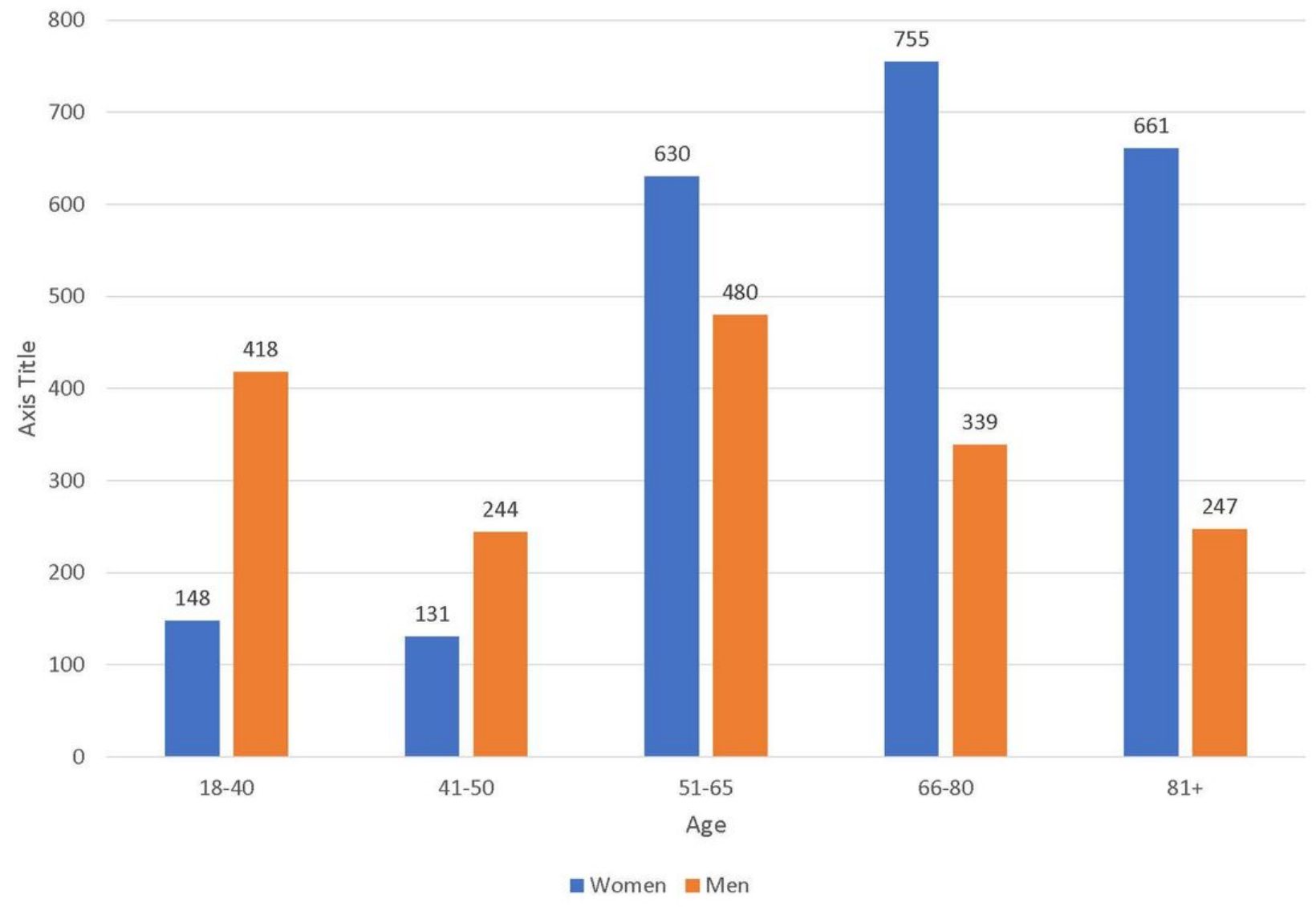

Figure 21

Multiple Shoulder Fractures 
Figure 22. Multiple radius and ulna Fractures in Ontario 2013-17

3000

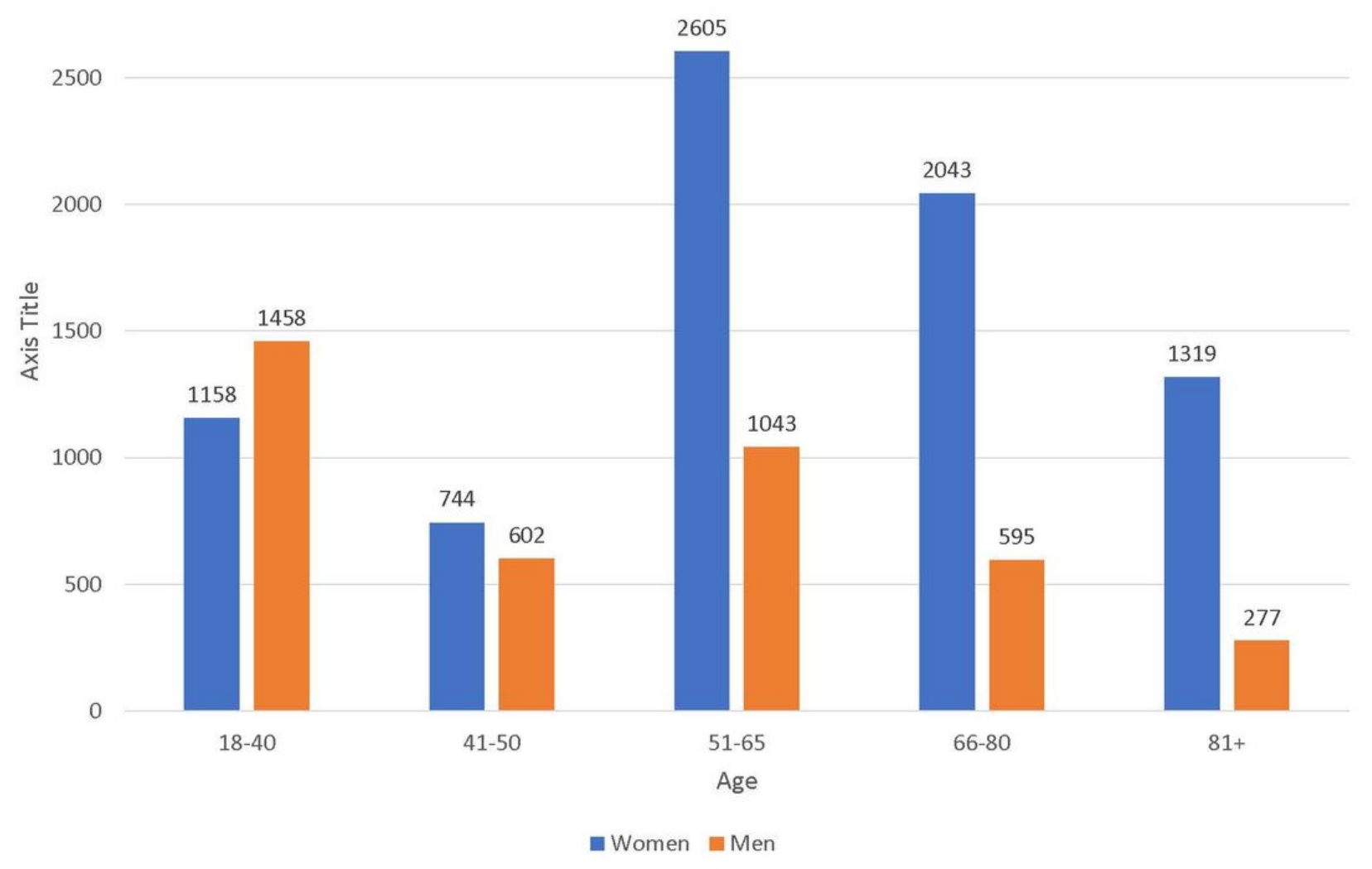

Figure 22

Multiple radius and ulna Fractures in Ontario 2013-17 
Figure 23. Percentage of men/women with diabetes by fracture type 35

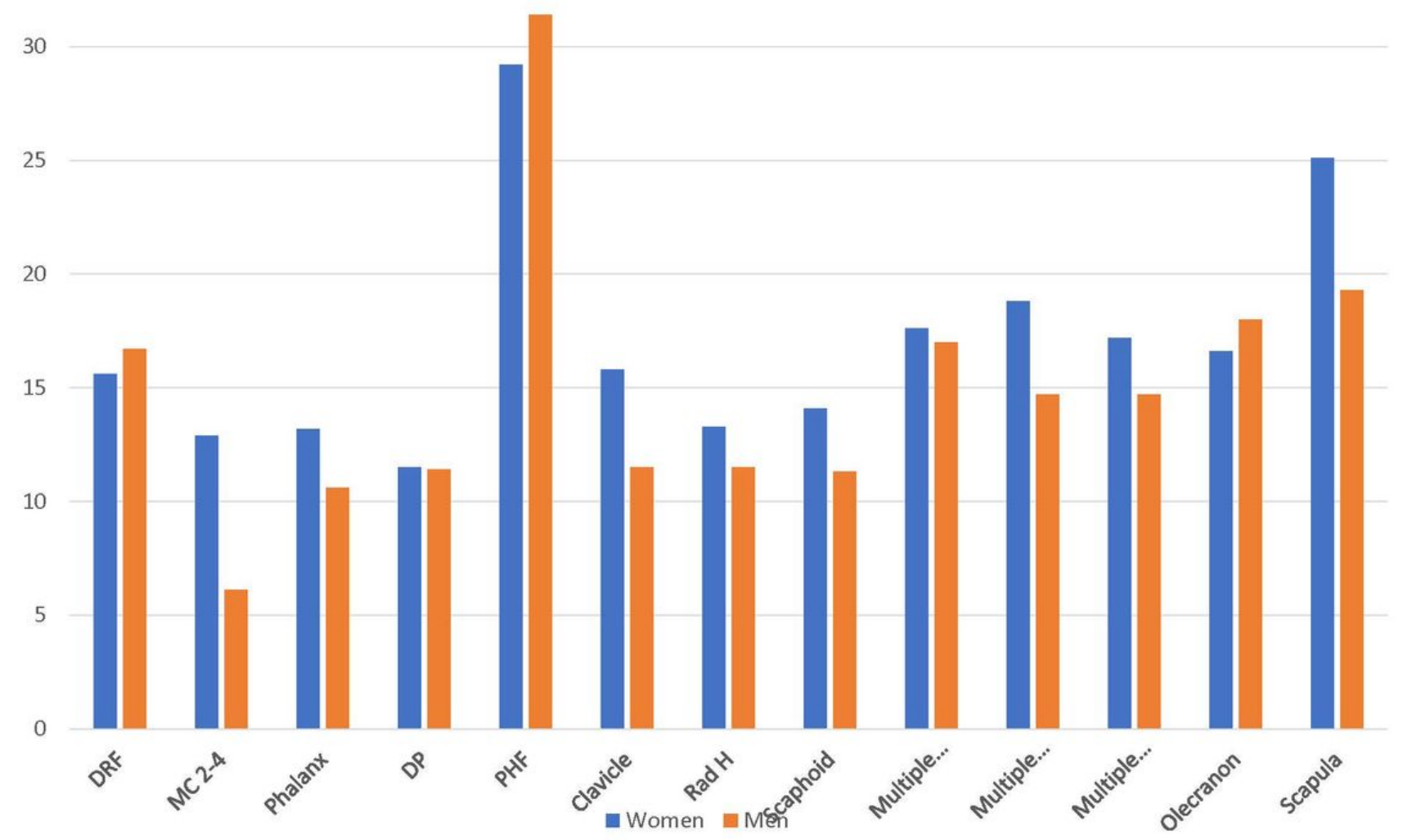

Figure 23

Percentage of men/women with diabetes by fracture type 
Figure 24. Percentage of men/women with RA by fracture type

6

4

3

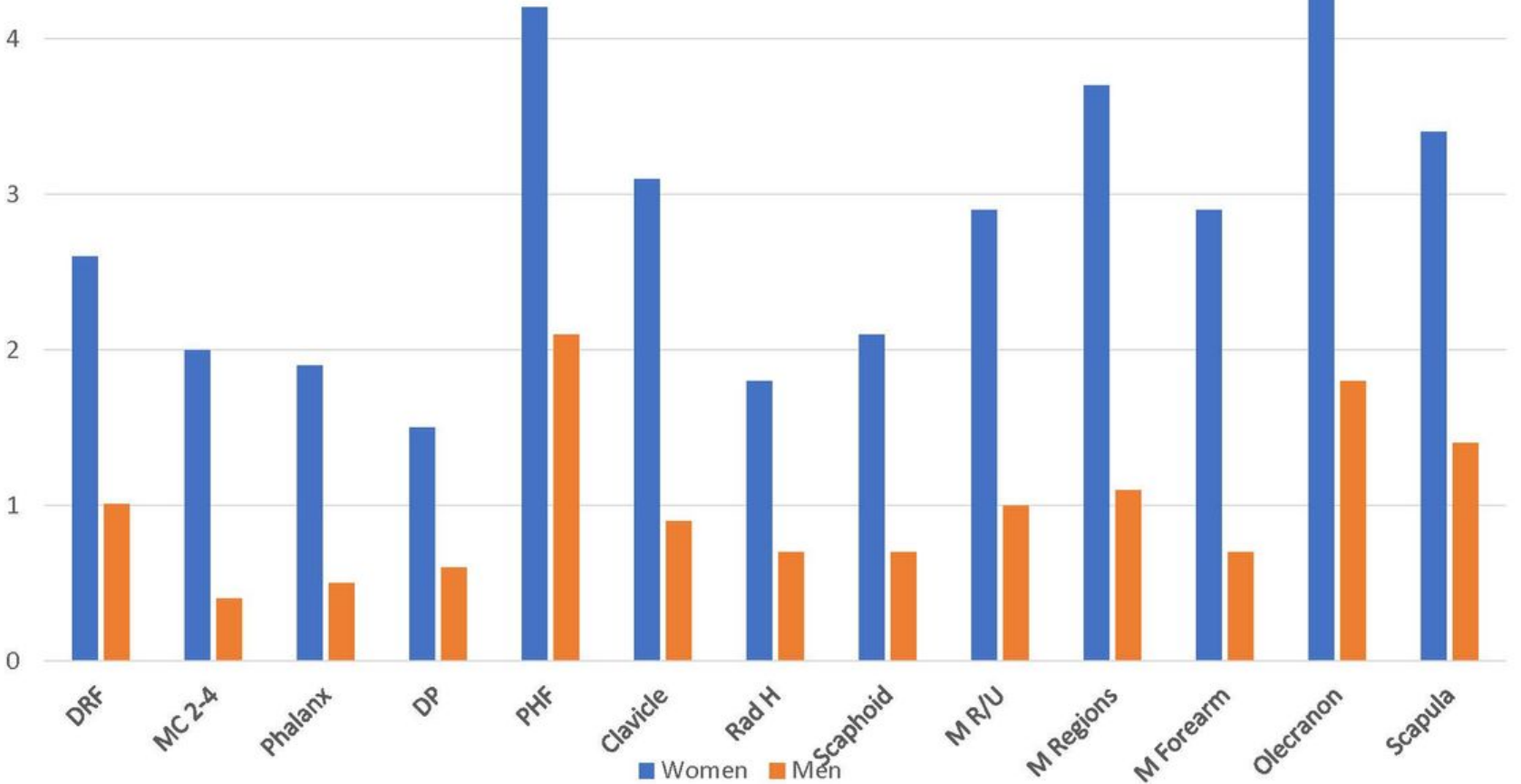

Figure 24

Percentage of men/women with RA by fracture type 


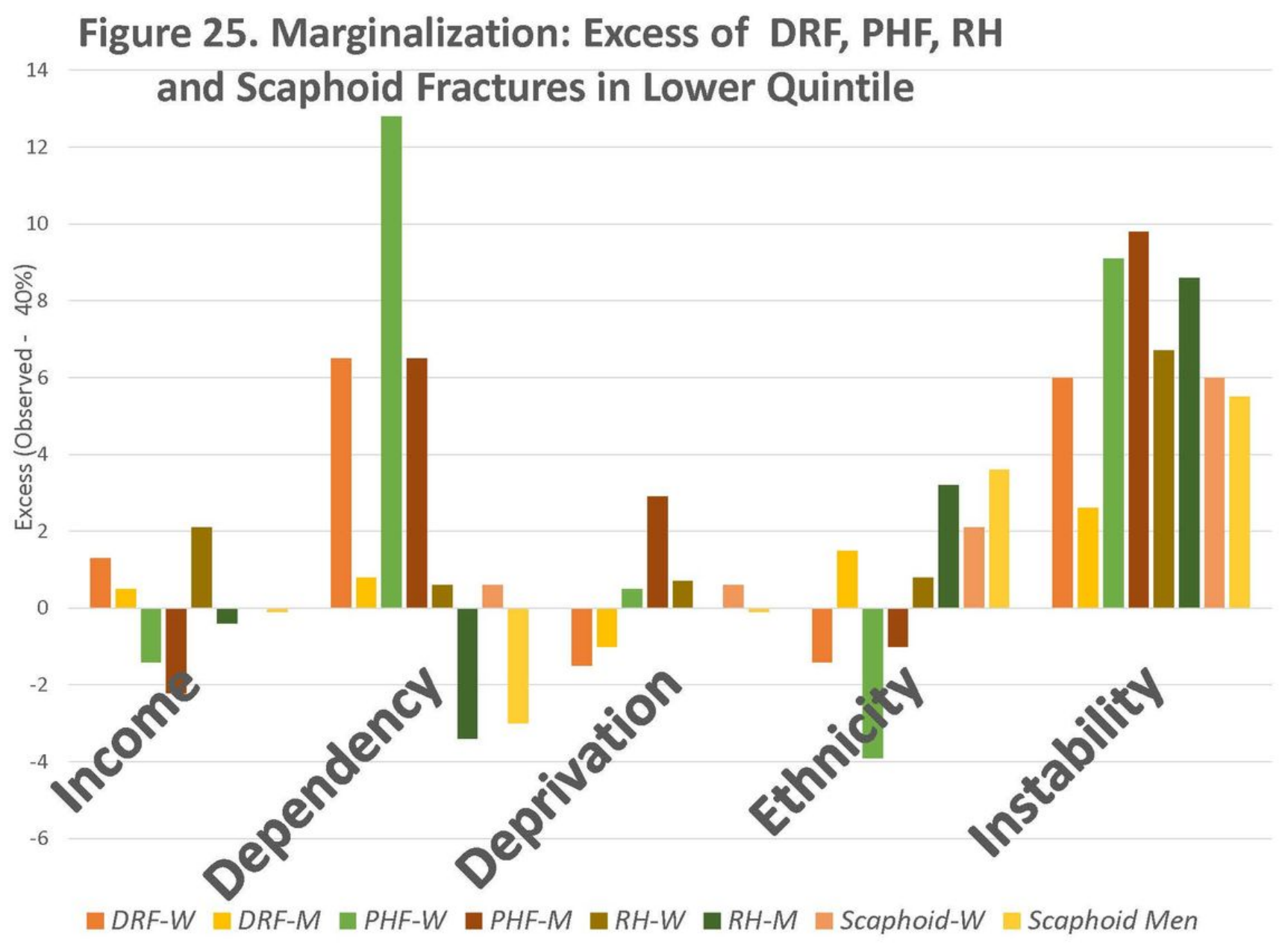

Figure 25

Marginalization: Excess of DRF, PHF, RH and Scaphoid Fractures in Lower Quintile 
Figure 26. Marginalization: Excess of Clavicle, Distal Phalanx, Metacarpal and Phalanx Fractures in Lower

\section{Quintile}

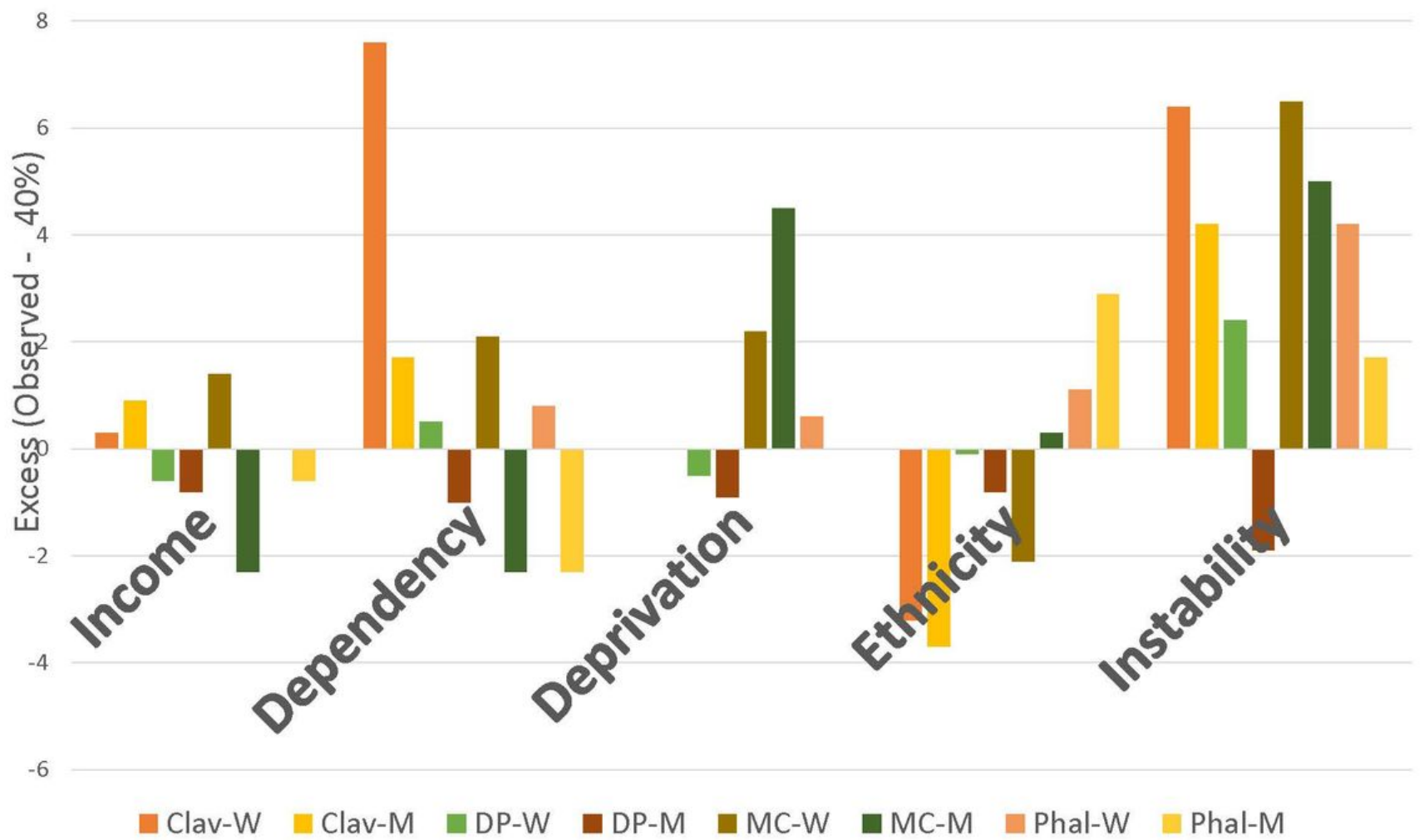

Figure 26

Marginalization: Excess of Clavicle, Distal Phalanx, Metacarpal and Phalanx Fractures in Lower Quintile 